\title{
The influence of solar wind on extratropical cyclones - Part 1: Wilcox effect revisited
}

\author{
P. Prikryl ${ }^{1}$, V. Rušin ${ }^{2}$, and M. Rybanský ${ }^{3}$ \\ ${ }^{1}$ Communications Research Centre Canada, Ottawa, ON, Canada \\ ${ }^{2}$ Astronomical Institute, Slovak Academy of Sciences, Tatranská Lomnica, Slovakia \\ ${ }^{3}$ Institute of Experimental Physics, Slovak Academy of Sciences, Košice, Slovakia
}

Received: 24 July 2007 - Revised: 19 August 2008 - Accepted: 22 October 2008 - Published: 6 January 2009

\begin{abstract}
A sun-weather correlation, namely the link between solar magnetic sector boundary passage (SBP) by the Earth and upper-level tropospheric vorticity area index (VAI), that was found by Wilcox et al. (1974) and shown to be statistically significant by Hines and Halevy (1977) is revisited. A minimum in the VAI one day after SBP followed by an increase a few days later was observed. Using the ECMWF ERA-40 re-analysis dataset for the original period from 1963 to 1973 and extending it to 2002, we have verified what has become known as the "Wilcox effect" for the Northern as well as the Southern Hemisphere winters. The effect persists through years of high and low volcanic aerosol loading except for the Northern Hemisphere at $500 \mathrm{mb}$, when the VAI minimum is weak during the low aerosol years after 1973, particularly for sector boundaries associated with south-to-north reversals of the interplanetary magnetic field (IMF) $B_{Z}$ component. The "disappearance" of the Wilcox effect was found previously by Tinsley et al. (1994) who suggested that enhanced stratospheric volcanic aerosols and changes in air-earth current density are necessary conditions for the effect. The present results indicate that the Wilcox effect does not require high aerosol loading to be detected. The results are corroborated by a correlation with coronal holes where the fast solar wind originates. Ground-based measurements of the green coronal emission line (Fe XIV, $530.3 \mathrm{~nm}$ ) are used in the superposed epoch analysis keyed by the times of sector boundary passage to show a one-toone correspondence between the mean VAI variations and coronal holes. The VAI is modulated by high-speed solar wind streams with a delay of 1-2 days. The Fourier spectra of VAI time series show peaks at periods similar to those found in the solar corona and solar wind time series. In the modulation of VAI by solar wind the IMF $B_{Z}$ seems to con-
\end{abstract}

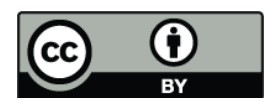

Correspondence to: P. Prikryl (paul.prikryl@crc.ca) trol the phase of the Wilcox effect and the depth of the VAI minimum. The mean VAI response to SBP associated with the north-to-south reversal of $B_{Z}$ is leading by up to 2 days the mean VAI response to SBP associated with the south-tonorth reversal of $B_{Z}$. For the latter, less geoeffective events, the VAI minimum deepens (with the above exception of the Northern Hemisphere low-aerosol 500-mb VAI) and the VAI maximum is delayed. The phase shift between the mean VAI responses obtained for these two subsets of SBP events may explain the reduced amplitude of the overall Wilcox effect.

In a companion paper, Prikryl et al. (2009) propose a new mechanism to explain the Wilcox effect, namely that solarwind-generated auroral atmospheric gravity waves (AGWs) influence the growth of extratropical cyclones. It is also observed that severe extratropical storms, explosive cyclogenesis and significant sea level pressure deepenings of extratropical storms tend to occur within a few days of the arrival of high-speed solar wind. These observations are discussed in the context of the proposed AGW mechanism as well as the previously suggested atmospheric electrical current (AEC) model (Tinsley et al., 1994), which requires the presence of stratospheric aerosols for a significant (Wilcox) effect.

Keywords. Meteorology and atmospheric dynamics (Convective processes; Mesoscale meteorology; Waves and tides)

\section{Introduction: solar wind influence on troposphere}

Possible influences of the solar wind and the ensuing geomagnetic activity on the winter mid-latitude troposphere on the scale of days have been suggested in the 1960s and 1970s. Wintertime deepening of $300-\mathrm{mb}$ troughs has been found to be statistically related to geomagnetic activity (McDonald and Roberts, 1960; Roberts and Olson, 1973). Atmospheric vorticity at the 300 - and 500-mb levels has been related to the solar magnetic sector structure (Wilcox et al., 1973,

Published by Copernicus Publications on behalf of the European Geosciences Union. 
1974), interplanetary interaction regions (Lundstedt, 1984), and large geomagnetic disturbances (Arora and Padgaonkar, 1981). Also, the high-latitude tropospheric pressure has been found to be correlated with the sector structure of the interplanetary magnetic field (Rostoker and Sharma, 1980). The vorticity area index (VAI) that was introduced by Roberts and Olson (1973) has been used in subsequent studies attempting to verify what has become known as the "Wilcox effect", namely a statistically significant decrease in VAI one day after solar magnetic sector boundary passage (SBP) by the Earth. The physical basis for the large-scale reversal in the interplanetary magnetic field (IMF) is a change in the heliospheric current sheet (HCS) structure (e.g. Hoeksema et al., 1983).

Larsen and Kelley (1977) studied observed and predicted meteorological indices and found a significant decrease in the accuracy of forecasts for the time period of approximately two days following a solar wind sector boundary crossing, thereby independently verifying the Wilcox effect. Other studies found either no effect or only a statistically insignificant effect (e.g. Bhatnagar and Jakobsson, 1978; Williams, 1978; Williams and Gerety, 1978; Burns et al., 1980). Nevertheless, the results by Wilcox et al. were thoroughly tested and found to be statistically significant (Hines and Halevy, 1977). In subsequent years, the effect seemed to disappear and reappear again twice, which was linked to stratospheric volcanic aerosols (Tinsley et al., 1994; Kirkland et al., 1996). These authors derived VAI from the National Meteorological Center (NMC) grids of 500-mb geopotential heights and found that correlations persisted for a few years following the Agung, El Chichón and Pinatubo volcanic eruptions, but were significantly weaker at other times. However, other studies found the effect during years of low aerosol concentration (Larsen and Kelley, 1977; Lundstedt, 1984). Also, the VAI appears to respond to Forbush decreases (FDs) of cosmic ray flux, and these are associated with most high speed solar wind streams (Tinsley et al., 1989; Tinsley and Deen, 1991). Thus, the presence of weak VAI responses to high speed streams in the absence of volcanic aerosols could be linked to FDs. The topic of sun-weather influences was reviewed by Taylor (1986), Tinsley and Deen (1991), Carslaw et al. (2002), Harrison and Carslaw (2003), and more recently was revisited by Burns et al. (2007).

Although general acceptance of the reality of a link between the solar wind and the troposphere has not been reached (partly because of a lack of an acceptable physical mechanism to explain the Wilcox effect), new results and important progress in understanding of a possible mechanism have been gained. In particular, Tinsley et al. (1994) suggest a mechanism that involves the global atmospheric electric circuit (AEC) and changes in relativistic electron precipitation linking the effects of charge accumulation to changes in ice nucleation. It has been shown that changes in the flux of precipitating relativistic electrons modulate the increased stratospheric resistivity due to the aerosols (Tins- ley et al., 1994; Kirkland et al., 1996; Kniveton and Tinsley, 2004; Roldugin and Tinsley, 2004; Tinsley and Zhou, 2006). Resulting changes in current density $J_{z}$ affect space charge (Zhou and Tinsley, 2007) and there are several cloud microphysical processes that could respond to space charge. These include electrically enhanced scavenging of aerosols that affects cloud lifetime, cloud cover, and precipitation (Tinsley et al., 1994). The mechanism invoking $J_{z}$ in the cloud changes have been supported by modelling and observational work (Tinsley, 2000; Tinsley at al., 2001, 2006, 2007; Tinsley and Zhou, 2006; Zhou and Tinsley, 2007; Burns et al., 2007). Of course, as pointed out by Burns et al. (2007), the mechanism invoking a role for $J_{z}$ in cloud changes does not exclude the possible contribution of changes in the galactic cosmic ray flux (Yu and Turco, 2001) that is modulated by solar wind and appears to be associated with VAI variations (Tinsley et al., 1989; Tinsley and Deen, 1991). Also, the AEC mechanism is invoked to explain the linear responses of atmospheric pressure to changes in polar cap ionospheric potential, under quiet conditions (Burns et al., 2007).

Prikryl et al. $(2001,2003)$ have suggested that auroral atmospheric gravity waves (AGWs) are another candidate for the "missing link" between the solar wind and tropospheric weather. Auroral AGWs may release instabilities that lead to tropospheric convection, convective clouds and storminess (Prikryl et al., 2009). Using the International Satellite Cloud Climatology Project (ISCCP) D1 dataset, a statistically significant response of high-level cloudiness to fast solar wind from coronal holes is found (Prikryl et al., 2003, 2006, 2009). These results are consistent with the previous finding of solar wind influence on mid-latitude tropospheric circulation (Wilcox et al., 1973; Lundstedt, 1984). In this paper, we use the improved meteorological reanalysis data to verify the Wilcox effect and to extend the analysis to the Southern Hemisphere. The results are corroborated by a correlation with coronal holes, from which high-speed solar wind streams flow. Also, the occurrence of severe extratropical weather events and extratropical storm sea level pressure deepenings is examined in the context of solar wind disturbances to support the argument that auroral AGWs may impact the extratropical cyclone activity after the arrival of high-speed solar wind streams.

\section{Instruments, techniques and data}

The European Centre for Medium-Range Weather Forecasts ERA-40 Reanalysis project (Uppala et al., 2005) consists of a number of climate datasets spanning the period September 1957 to August 2002 (http://data.ecmwf.int/data/). The 12hourly time series of the relative vorticity at the 500- and 300-mb levels (at 00:00 and 12:00 UTC for each day) are extracted and converted to absolute vorticity to compute VAI.

The National Space Science Data Center (NSSDC) OMNIWeb http://omniweb.gsfc.nasa.gov provides the solar wind 
plasma and interplanetary magnetic field (IMF) database that is assembled from observations starting in 1963 by many spacecraft - primarily ISEE and IMP series, Wind and ACE. Some plasma data gaps are filled with data from the Proton Monitor on the Solar and Heliospheric Observatory (SOHO).

Intensity measurements since 1939 of the green coronal emission line (Fe XIV, $530.3 \mathrm{~nm}$ ) were obtained by several ground-based coronagraphs and have been merged into a homogeneous coronal dataset based on the Lomnický Štít photometric scale since 1965 (Rybanský, 1975; Rybanský et al., 2001, 2005). The coronal emission data were processed and coronal index derived at the Astronomical Institute of Slovak Academy of Sciences. The coronal intensities measured at a height of 40 arcseconds above the solar limb are expressed in absolute coronal units (ACU) (ftp: //ftp.ngdc.noaa.gov/stp/solar_data). One ACU represents the intensity of the continuous spectrum from the center of the solar disk with a width of $1 \AA$ at the same wavelength as the observational spectral line $\left(1 \mathrm{ACU}=3.89 \mathrm{~W} \mathrm{~m}^{-2} \mathrm{sr}^{-1}\right.$ at $530.3 \mathrm{~nm}$ ). Synoptic charts of the green corona intensity at the solar central meridian are constructed from these data from 1963 to 2005. Recently, Rušin and Rybanský (2002) showed that the green line intensity and photospheric magnetic fields were highly correlated from 1976 to 1999 , so the higher the coronal intensity, the stronger the magnetic field.

The Atlas of Extratropical Storm Tracks from 1961 to 1998 (Chandler and Jonas, 1999) that is available on-line (http://www.giss.nasa.gov/data/stormtracks) is derived from the National Centers for Environmental Predictions/ National Center for Atmospheric Research (NCEP/NCAR) Reanalysis project.

\section{High-speed plasma streams from coronal holes}

The solar wind is an "extension of the solar corona into interplanetary space" (Parker, 1959). Because the solar magnetic field defines the structure of the corona, the solar wind is structured accordingly into alternating sectors of fast and slow solar wind threaded by the solar magnetic field that is dragged outward forming the interplanetary magnetic field (IMF), forming Parker spirals. The IMF reverses its polarity at magnetic sector boundaries that usually precede corotating interaction regions (CIRs). This fairly regular and recurrent solar wind structure can be traced back to the solar corona although it is sometimes disrupted by coronal mass ejections (CMEs).

A CIR between the fast and slow solar wind is characterized by enhanced magnetic field magnitude, plasma density and temperature (Siscoe, 1972; Rosenberg and Coleman, 1980). As the CIR passes by a spacecraft the solar wind density $n_{p}$ rises to a maximum and then drops sharply within a narrow interface while the magnetic field magnitude $|\mathbf{B}|$, the plasma temperature and solar wind velocity $V$ increase (Belcher and Davis, 1971; Burlaga, 1974; Gosling et al.,
1978). The mean standard deviation $\sigma_{B z}$ of the IMF $B_{Z}$ component also increases within the CIR and past the stream interface.

It is advantageous to combine some of the parameters in a single variable that would characterize the solar wind geoeffectiveness and correlate with solar activity indices. Osherovich et al. (1999) suggested a quasi-invariant in$\operatorname{dex} \mathrm{QI} \equiv\left(B^{2} / 8 \pi\right) /\left(\rho V^{2} / 2\right)=M_{A}^{-2}$, where $\rho$ is plasma density (protons in the first approximation), $M_{A}$ is the magnetic Mach number $\left(M_{A}=V / V_{A}\right.$, where $V_{A}$ is the Alfvén speed) and $B=|\mathbf{B}|$. The index is equivalent to the ratio of the solarwind magnetic to ram pressures, or the inverse of the magnetic Mach number squared. This non-dimensional quantity is of fundamental importance for describing the solar wind.

The interface is the leading edge of a high-speed solar wind plasma stream (hence referred to as plasma stream or simply stream) where the amplitudes of solar wind Alfvén waves increase abruptly (Belcher and Davis, 1971). Although the amplitudes of Alfvén wave trains diminish as the solar wind velocity decreases, they are a source of highintensity, long-duration continuous auroral activity (Tsurutani and Gonzales, 1987). Lundstedt (1984) shows that there is a strong response of the auroral electrojet (AE) and geomagnetic indices to the steep increase of the solar wind power input across the CIR interface. It is expected that the energy transferred to the neutral atmosphere as the result of Joule heating or Lorentz forcing and the ensuing gravity waves likewise increases.

As a consequence of solar rotation and coronal holes, CIRs along with plasma streams are recurrent solar wind structures that are usually separated by IMF sector boundaries and are most periodic during the declining phase of the solar cycle. On average, solar wind velocity reaches a maximum 2 days after the arrival of CIRs while the solar wind MHD waves have the largest amplitudes for about 2 days after the arrival of CIRs (Prikryl et al., 2009). In comparison, coronal mass ejections (CMEs) which are usually associated with shocks and solar plasma ejecta of low proton temperature (Richardson and Cane, 1995), or magnetic clouds displaying a characteristic helical structure, occur more frequently during the maximum of the solar activity. Similar to solar wind Alfvén waves, the compressional MHD waves associated with the CME shock couple to the magnetosphere, causing ionospheric convection enhancements, which in turn generate AGWs. The CME effects on the magnetosphereionosphere system are usually very intense but short-lived, with their intensity and duration depending on the IMF direction and the CME speed. In this paper, we refer to interplanetary CMEs observed in the solar wind plasma near the earth.

Figure 1a shows hourly averages of solar wind plasma parameters for a CIR event (Case 2 discussed by Prikryl et al., 2009) that displayed a rather sharp interface between the moderately enhanced and the slow solar wind on 15 November. The magnetic sector boundary approximately coincided 


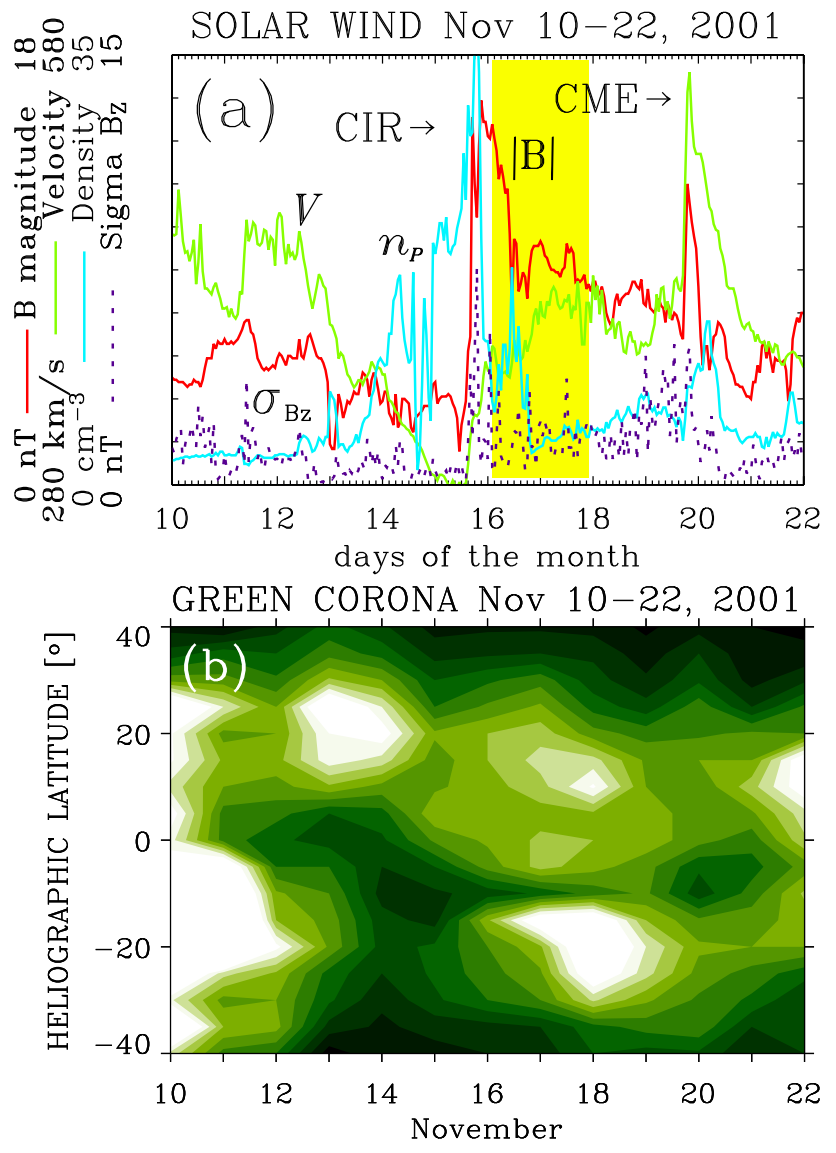

Fig. 1. (a) Solar wind velocity, density, magnetic field magnitude and standard deviation of the IMF $B_{Z}$ before and after the arrival of a corotating interaction region (CIR) on 15 November 2001. The magnetic sector boundary coincided with the stream interface at 17:50 UT. A coronal mass ejection (CME) arrived on November 19. The yellow shaded area indicates approximate time interval of a rapid cyclogenesis in the Gulf of Alaska. (b) A Synoptic chart of the green corona interpolated from the 530.3-nm emission measurements on the east and west solar limb. Darker color represents lower intensity and contours are 5 ACUs apart.

with the stream interface that passed by ACE spacecraft at 17:50 UT. In the compression region of the CIR at the leading edge of the fast solar wind, the density $n_{p}$ rises to maximum and then falls while $|\mathbf{B}|$ peaks sharply. In comparison, all three plasma parameters $\left(V,|\mathbf{B}|\right.$ and $\left.n_{p}\right)$ increased sharply across the CME shock that arrived 4 days later on 19 November. The IMF $\sigma_{B z}$ is enhanced both before and after the CME shock because the Alfvén waves in the stream interact with the shock (see, e.g. references in Prikryl et al., 2005). The enhanced $\sigma_{B z}$ indicates enhanced amplitudes of the IMF fluctuations (MHD waves) which couple to the magnetosphere and pulse the ionospheric convection, resulting in auroral AGWs (Prikryl et al., 2009).

\section{The VAI response to solar corona and/or solar wind structure}

We use the ground-based measurements of the green coronal emission (Fe XIV, $530.3 \mathrm{~nm}$ ) at the solar limb to construct synoptic charts of the coronal intensity at the solar central meridian. For a given day, the central meridian intensity is approximated by the average of the intensities measured at the east limb approximately $1 / 4$ of the synodic solar rotation, or 7 days earlier, and at the west limb 7 days later. Synoptic charts of the green corona are used to identify the lowemission regions (coronal holes), particularly those near the heliographic equator. The geomagnetic and atmospheric responses to a coronal hole passing the central heliographic meridian are observed 2-4 days later. Figure 1 b shows the green corona synoptic map for a 12-day period around the day of sector boundary crossing at $\sim 00: 00$ UT and a CIR at 17:50 UT on 15 November (Fig. 1a). A moderate solar wind stream that followed originated from the coronal hole that crossed the solar central meridian on 14 November. Following the arrival of the CIR an extratropical cyclone evolved over a 2-day period and displayed a series of mesoscale bands (Prikryl et al., 2009).

\subsection{Verification of the 500-mb VAI superposed epoch analysis results (1963-1973)}

Wilcox et al. (1974; their Fig. 1) found the VAI response to solar wind structure, namely a dip in VAI about one day after sector boundary passage (SBP) as reproduced in Fig. 2a (line plot). The superposed epoch (SPE) analysis (e.g. Ambrož, 1979) of 12-hourly VAI time series for the 500-mb level used 131 SBP events during winter months of November-March for period of 1963-1973. The error bar indicates the standard error of the mean $\left( \pm 0.5 \sigma_{M}\right)$ (Wilcox et al., 1974). In a similar analysis, Lundstedt (1984) found the VAI minimum to occur near the arrival of stream-interaction regions at the leading edge of high-speed solar wind. The IMF sector boundaries, if occurring in the vicinity of CIRs, generally precede the interaction regions (stream interfaces) by up to about $1 \frac{1}{2}$ days (Gosling et al., 1978). While focusing on the VAI minimum, both studies showed that the mean VAI reached a maximum 3-4 days after the SBP and was preceded by a smaller maximum before the SBP day. As discussed below, the VAI variation is a delayed response to a weakening solar wind that is being replaced by the next plasma stream (Fig. 2b).

For comparison, the SPE analysis result for the green corona using 131 SBP events is shown in Fig. 2a. The original list of 131 SBP events used by Wilcox et al. was not available. We have used solar wind OMNI data in conjunction with the IMF polarity inferred from ground-based magnetometers (Svalgaard, 1975, 2006, private communication) to identify the SBP events. The dates and times of these events, which may differ from those that were identified by Wilcox et al. (1974), are used in the SPE analysis of daily 
solar corona intensity, hourly solar wind plasma parameters and 12-hourly VAI time series. Table 1 lists SBP events from 1963 to August 2002 used in this analysis.

Figure 2a shows the mean central meridian coronal intensity before and after the sector boundary passage. The mean coronal hole is found centered on day -1 . Figure $2 b$ shows the corresponding averaged solar wind data with representative standard error bars, i.e. \pm 1 standard error of the mean $\left(\sigma_{M}\right)$. A clear signature of superposed CIRs just after day 0 at the leading edge of the mean high-speed plasma stream is found. This means that, on average, SBPs closely precede and sometimes are coincident with CIR interfaces.

The mean solar wind speed has a minimum near day -1 and ramps up just after day 0 towards a maximum a couple of days later. Thus the mean CIR and the stream interface map back to the leading edge of the mean coronal hole just past day 0 (Fig. 2a). The mean CIR does not show the stream interface as sharply as the event shown in Fig. 1b and is relatively broad because the sector boundary usually does not coincide with the stream interface. It is noted that the mean standard deviation $\sigma_{B z}$, which is a measure of solar wind Alfvén wave amplitude, is strongly enhanced for a few days after the SBP.

Wilcox et al. (1976, their Fig. 4) extended the SPE analysis of VAI to a wide epoch period of \pm 180 days to show that the dip in VAI near day +1 is the largest and therefore statistically significant. Their result for 1963-1973 is reproduced for \pm 30 days in Fig. 3a showing the 500-mb VAI variation superposed on the mean green corona intensity. It is observed that a series of mean coronal holes 7-10 days apart is closely associated with the VAI oscillations as well as the mean solar wind speed variations due to corotating streams (Fig. 3b).

As in Fig. 2b, the averaged solar wind parameters in Fig. 3b show a clear signature of superposed CIRs near day 0 at the leading edge of the mean high-speed plasma stream associated with the central mean coronal hole. While the main Wilcox pattern (VAI minimum) is associated with the primary coronal hole, there is a one-to-one correspondence between the secondary VAI minima/maxima and the mean coronal holes before and after the key day. The solar wind data show the corresponding averaged streams and CIRs; the key stream interface at day 0 being the sharpest. There is a one-to-one correspondence between the minima/maxima of the solar wind speed and the VAI minima/maxima, although the mean streams and CIRs that are associated with the two shallow mean coronal holes near -20 and +7 days are smeared out and relatively weak. The deepest minima of the mean solar wind velocity $V$ at $-28,-1$ and +27 days preceded the VAI minima by about 1 day.

Another solar wind plasma parameter needs to be considered, namely the IMF $B_{Z}$ component (in geocentric solar magnetospheric (GSM) coordinate system) that controls the coupling to the magnetosphere and the amount of joule heating energy deposition in the auroral ionosphere. Because the IMF $B_{Z}$ changes sign frequently, particularly when large am-
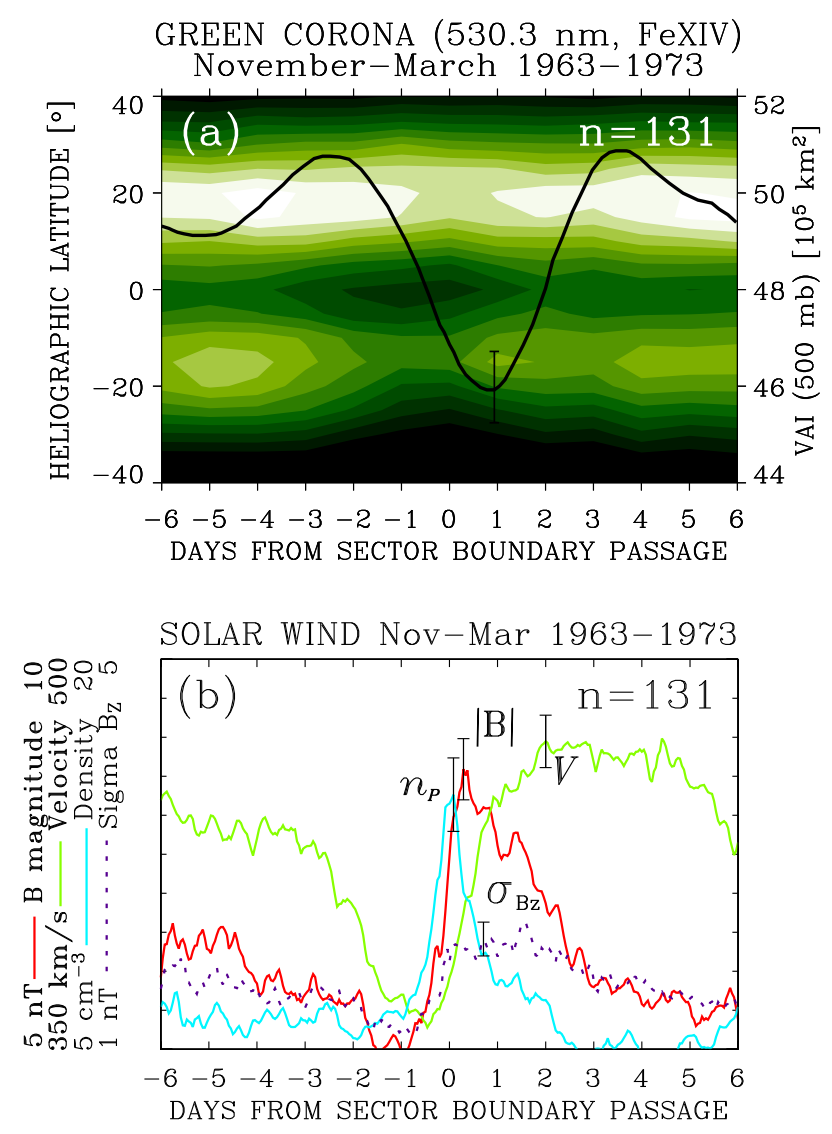

Fig. 2. The superposed epoch analysis of (a) the green solar corona emission line intensity interpolated for the central meridian and (b) solar wind plasma parameters. Representative error bars plotted for each solar wind plasma parameter are twice the standard error of the mean. All 131 12-day long time series are keyed by the magnetic sector boundary passage that occurred during the northern "winter" of November-March from 1963 to 1973. The intensity contours are 2 ACUs apart. The VAI result by Wilcox et al. (1975, their Fig. 1) is superposed in the top panel.

plitude Alfvén waves are present in the solar wind, when it is averaged over all SBP events the mean IMF $B_{Z}$ component shows only small amplitude fluctuations about zero and no significant trend around the key day (not shown in Fig. 3c). However, CIRs can be classified by IMF polarity when considering the Russell-McPherron effect (Russell and McPherron, 1973) for months around the spring and fall equinoxes, as shown by Miyoshi and Kataoka (2008). The mean IMF $B_{Z}$ component for one group of events, which the latter authors denote SBZ (southward $B_{Z}$ ), systematically shifts southward near the CIR interface while the NBZ (northward $B_{Z}$ ) group of CIRs shows a northward shift of the IMF $B_{Z}$. Strictly speaking, to subdivide our dataset of SBP events in such a way it is not possible to follow exactly the same rule that was applied by the latter authors, because we are interested in winter (cold season) months as selected above. 
Table 1. List of times (yymmddhh) of magnetic sector boundary crossings of the IMF toward(-)/away(+).

\begin{tabular}{|c|c|c|c|c|c|c|}
\hline $63011300-/+$ & $65061412+/-$ & $68071000+/-$ & $71061600-/+$ & $74062603-/+$ & $77042415-/+$ & $80072700-/+$ \\
\hline 63012900+/- & $65062503-/+$ & $68071712-/+$ & $71062818+/-$ & $74070600+/-$ & $77042900+/-$ & $80080215+/-$ \\
\hline $63021000-/+$ & $65062903+/ /-$ & $68072512+/-$ & $71071212-/+$ & $74072300-/ /+$ & $77051203+/-$ & $80091312+/-$ \\
\hline $63030800+/-$ & $65070600-/+$ & $68073120-/+$ & $71071815+/-$ & $74080215+/-$ & $77052100-/+$ & $80092212-/+$ \\
\hline $63041100+/-$ & $65072300-/+$ & $68080512+/-$ & $71072618+/-$ & $74081906-/+$ & $77060806+/-$ & $80092800+/-$ \\
\hline $63041900-/+$ & $65072712+/-$ & $68081400-/+$ & $71080810-/+$ & $74082912+/-$ & $77061615-/+$ & $80101800-/+$ \\
\hline $63042300+/-$ & $65081812-/+$ & $68082103+/-$ & $71082300+/-$ & $74091215-/+$ & $77062800+/-$ & $80102312+/-$ \\
\hline $63042800-1+$ & $65082314+/-$ & $68090212-/+$ & $71090422-/+$ & $74092406+1-$ & $77070109-/+$ & $80103100-/+$ \\
\hline $63050800+/-$ & $65091606-/+$ & $68091000-/+$ & $71091312+/-$ & $74101300-/+$ & $77070600+/-$ & $80110415+/-$ \\
\hline $63051700-/+$ & $65092212+/-$ & $68091900+/-$ & $71093009-/+$ & $74102300+/-$ & $77071406-/+$ & $80111506-/+$ \\
\hline $63052800-/+$ & $65102606+/-$ & $68100700-/+$ & $71101212+/-$ & $74110820-/+$ & $77072712+/-$ & $80112018+/-$ \\
\hline $63060700+/-$ & $65111812-/+$ & 68101614+/- & $71102720-/+$ & $74111909+/-$ & $77080312+/-$ & $80112600-/+$ \\
\hline $63061400-/+$ & $65112400+/-$ & $68111308+/-$ & $71110412+/-$ & $74120712-/+$ & $77081200-/+$ & $80113000+/-$ \\
\hline $63062500-/+$ & $65113008-/+$ & $68112920-/+$ & $71112212-/+$ & $74121700+/-$ & $77083103+/-$ & $80121200-/+$ \\
\hline $63070400+/-$ & $65120408+/ /-$ & $68121000+/-$ & $71120100+/-$ & $74122600-/+$ & $77090900-/+$ & $80123100+/-$ \\
\hline $63072100-/+$ & $65121610-/+$ & $68122421-/+$ & $71121800-/+$ & $74123000+/-$ & $77092622+/-$ & $81011009-/+$ \\
\hline $63073000+/-$ & $65122203+/-$ & $69010618+/-$ & $71122800+/-$ & $75010312-/+$ & $77101100-/++$ & $81012209-/+$ \\
\hline $63081800-/+$ & $66010200+/-$ & $69012400-/+$ & $72011600-/+$ & $75011218+/-$ & $77102500+/-$ & $81012618+/-$ \\
\hline $63082500+/-$ & $66011412-/+$ & $69020216+/-$ & $72012112+/-$ & $75012302-/+$ & $77110415-/+$ & $81020800-/+$ \\
\hline $63091400-/+$ & $66012010+/-$ & $69021721-/++$ & $72021012-/+$ & $75012702+/-$ & $77112000+/-$ & $81022215+/-$ \\
\hline $63092100+/-$ & $66020300+/-$ & $69030508+/-$ & $72021710+/-$ & $75013100-/+$ & $77121021-/+$ & $81030412-/+$ \\
\hline $63101000-/+$ & $66021200-/++$ & $69032410-/+$ & $72030700-/+$ & $75020815+/-$ & $77122403+/-$ & $81032500+/-$ \\
\hline $63102200+/-$ & $66030312+/-$ & $69033118+/-$ & $72031600+/-$ & $75021906-/+$ & $78012600+/-$ & $81040412-/+$ \\
\hline $63110400-/+$ & $66030800-/+$ & $69042100-/+$ & $72040400-/+$ & $75030800+/-$ & $78020818-/+$ & $81041815+/-$ \\
\hline $63111700+/-$ & $66033000+/-$ & $69042800+/-$ & $72041012+/-$ & $75032100-/+$ & $78021500+/-$ & $81043009-/+$ \\
\hline $63120300-/+$ & $66042800+/-$ & $69050806-1+$ & $72050900+/-$ & $75040300+/-$ & $15-/+$ & $81051503+/-$ \\
\hline $63121300+/-$ & $66051000-/+$ & $69051300+/-$ & $72052806-/+$ & $75041700-/+$ & $78022600+/-$ & $81052800-/+$ \\
\hline $63122000-/+$ & $66051600+/-$ & $69051900-/+$ & $72060400+/-$ & $75050109+/-$ & $00-/+$ & $81061418+/-$ \\
\hline $63122800+/-$ & $66052100-/+$ & $69052800+/-$ & $72062418-/+$ & $75051603-/+$ & $78031500+/-$ & $81062218-/+$ \\
\hline $64010112-/+$ & $66052600+/-$ & $69061418-/+$ & $72070300+/-$ & $75052700+/-$ & $78032300-/+$ & $81071103+/-$ \\
\hline $64010800+/-$ & $66060200-/+$ & $69062303+/-$ & $72071109-/+$ & $75061103-/+$ & & $81072012-/+$ \\
\hline $64011606-/+$ & $66061100+/-$ & $69071112-/+$ & $72071600+/-$ & $75062700+/-$ & $78041200+/-$ & $81080721+/-$ \\
\hline $64012309+/-$ & $66061712-/++$ & $69072200+/-$ & $72072300-/+$ & $75070803-/+$ & $78041712-/+$ & $81081700-/+$ \\
\hline $64012800-/+$ & $66062300+/-$ & $69080712-/+$ & $72080312+/-$ & $75072500+/-$ & $78042300+/-$ & $81090300+/-$ \\
\hline $64020406+/-$ & $66070800+/-$ & $69081712+/-$ & $72081700-/+$ & $75080104-/+$ & $78050700-/+$ & $81091109-/+$ \\
\hline $64021200-/+$ & $66071518-/+$ & $69090508-1+$ & $72090400+/-$ & $75082015+/-$ & $78051100+/-$ & $81091900+/-$ \\
\hline $64022012+/-$ & $66072008+1-$ & $69091700+/-$ & $72090810-/+$ & $75082900-/+$ & $18+/-$ & $81092406-/+$ \\
\hline $64030400+/-$ & $66080900-/+$ & $69101606+/-$ & 721001 & $00+/-$ & $78060212+/-$ & $81092908+/-$ \\
\hline $100-/+$ & $66081810+/-$ & $69103100-/+$ & $00-/+$ & $02-1+$ & $12-/+$ & $81100806-/+$ \\
\hline $64033100+/-$ & $66090600-/+$ & $69112615-/+$ & $72103015+/-$ & 75101415+/- & $78061900+/-$ & $81102606+/-$ \\
\hline $64040800-/+$ & $66091412+/-$ & $69120900+/-$ & $72110616-/+$ & $75102900-/+$ & $02-1+$ & $81110500-/+$ \\
\hline $64042300+/-$ & $66100412-/+$ & $9122222-/+$ & $300+/-$ & $75110910+/-$ & $78081410+/-$ & $81112415-/+$ \\
\hline $64050500-/+$ & $66101203+/-$ & $70010500+/-$ & $72121300-/+$ & $75112500-/+$ & $78082503-/+$ & $811201115-/+$ \\
\hline $64052400+/-$ & $66103012-/+$ & $70011900-/+$ & $72122200+/-$ & $75120800+/-$ & $78091300+/-$ & $81121003+/-$ \\
\hline $64053100-/+$ & $66110800+/-$ & $70020112+/-$ & $73010700-/+$ & $75122500-/+$ & $78092115-/+$ & $81121718-/+$ \\
\hline $64060600+/-$ & $66112721-/+$ & $70020921-/+$ & $73011900+/-$ & $76010300+/-$ & $78100900+/-$ & $81122221+/-$ \\
\hline $64061000-/+$ & $66120408+/ /-$ & $70022800+/-$ & $00-/+$ & $912-/+$ & $00-/+$ & $82010621+/-$ \\
\hline $64061800+/-$ & $66122400-/+$ & $70030821-/+$ & $73021618+/-$ & $76013100+/-$ & $78110706+/-$ & $82011418-/+$ \\
\hline $64062700-/+$ & $67010115+/-$ & $5+/-$ & $0-1+$ & $10-/+$ & $8+/-$ & $82021503-/+$ \\
\hline & $67010606-/+$ & $00-/+$ & $0+/-$ & $0+1-$ & $2-1+$ & $82030612+/-$ \\
\hline $64070700-/+$ & $67011306+/-$ & $70051118+/-$ & $20-/+$ & $76031500-/+$ & $78122800+/-$ & $82031800-/+$ \\
\hline $64071700+/-$ & $67011810-/+$ & $2800-/+$ & $8+1-$ & $00+/-$ & $0-1+$ & $2903+/-$ \\
\hline $64072900+/-$ & $67020700+/-$ & $70060718+/-$ & $900-/+$ & $76041000-/+$ & $0+1-$ & $82041100-/+$ \\
\hline $64080300-/+$ & $67022300-/+$ & $70062400-/+$ & $73051209+/-$ & $76042200+/-$ & $03-1+$ & $82042800+/-$ \\
\hline & $67031900+/-$ & $70070400+/-$ & $73052800-/+$ & $76050806-/+$ & $0+1-$ & $82050903-1+$ \\
\hline $64082500+/-$ & $67032300-/+$ & $70080112+/-$ & $73061010+/-$ & 76051912+/- & $00-1+$ & $82052600+/-$ \\
\hline $64083100-/+$ & $67040400+/-$ & $1600-/+$ & $6-1+$ & $316-/+$ & $\theta+1-$ & $82060512-/+$ \\
\hline & & & & & $9+1-$ & $2300+/-$ \\
\hline $64091700-/+$ & $67051100+/-$ & $6-1+$ & $90+1-$ & $0+1-$ & $18-/+$ & $82070500-/+$ \\
\hline $64092200+/-$ & $67071100+/-$ & $2+1-$ & $0+/-$ & $9-/+$ & $9+/-$ & $12+/-$ \\
\hline $64092712-/+$ & $67080412-/+$ & $70101100-/+$ & $12-/+$ & $1800-/+$ & $12-/+$ & $82073100-/+$ \\
\hline $64100312+/-$ & $67080900+/-$ & $70102700+/-$ & $00+/-$ & $600+/-$ & $99+/-$ & $82081600+/-$ \\
\hline 641012 & 670831 & $2-1+$ & $0-1+$ & $0-1+$ & $06-1+$ & $82082815-/+$ \\
\hline $64101800+/-$ & $67090616+/-$ & $70112410+/-$ & $73100812+/-$ & $500-1+$ & $79071202+/-$ & $82090812+/-$ \\
\hline $64102600-/+$ & $67092710-/+$ & & $73101600-/+$ & $400+/-$ & $79072800-/+$ & $82100821+/-$ \\
\hline $64110100+/-$ & $67100306+/-$ & $9+1-$ & & $8-/+$ & $21+/-$ & $82102900-/+$ \\
\hline $64110800-/+$ & $67102512-/+$ & $9-1+$ & $0-1+$ & $9+/-$ & $2-1+$ & 8211 \\
\hline $64111500+/-$ & 671121 & $5+/-$ & $8+/-$ & $0-1+$ & & $18-/+$ \\
\hline & $67120500+/-$ & $000-/+$ & $2-1+$ & & $00-/+$ & \\
\hline $64112700+/-$ & $67121606-/+$ & $808+/-$ & $2+1-$ & $2-1+$ & $00+/-$ & $700+/-$ \\
\hline $64120612-/+$ & $68010200+/-$ & & $2-1+$ & $76120200+/-$ & $18-/+$ & $83010206-/+$ \\
\hline $64121400-/+$ & $68011612-/+$ & $71021403+/-$ & & $76120809-/+$ & & $83012400+/-$ \\
\hline $64122600+/-$ & $68012900+/-$ & $71022400-/+$ & $12-/+$ & $76121600+/-$ & $00-/+$ & $83012912-/+$ \\
\hline & $68021218-/+$ & $71030200+/-$ & $74012412+/-$ & $76122112-/+$ & $80012715+/-$ & $83020300+/-$ \\
\hline $65010800+/-$ & $68022700+/-$ & $71030800-/+$ & $74021100-/+$ & $76123000+/-$ & $80020306-/+$ & $83021100-/+$ \\
\hline & $68031012-/+$ & & & $77010506-/+$ & & $83021918+/-$ \\
\hline $65020400+/-$ & $68032315+/-$ & $71032300-/+$ & $74030903-/+$ & $77011100+/-$ & $80030312-/+$ & $83030915-/+$ \\
\hline & $68040520-/+$ & $71033000+/-$ & & $77011900-/+$ & $80042512-/+$ & $83031812+/-$ \\
\hline $65050500+/-$ & $68042110+/-$ & $71040403-/+$ & $74040312-/+$ & $77012600+/-$ & $80050506+/-$ & $83040500-/+$ \\
\hline $65051500-/+$ & $68050200-/+$ & $71040812+/-$ & $74041606+/-$ & $77020109-/+$ & $80060700+/-$ & $83041300+/-$ \\
\hline $65052100+/-$ & $68051714+/-$ & $71050518+/-$ & $74050215-/+$ & $77020500+/-$ & $80061409-/+$ & $83042400+/-$ \\
\hline $65052700-/+$ & $68052912-/+$ & $71051706-/+$ & $74051315+/-$ & $77022800-/+$ & $80062518+/-$ & $83050200-/+$ \\
\hline $65060100+/-$ & $68060706-/+$ & $71052300+/-$ & $74053003-/+$ & $77030812+/-$ & $80071115-/+$ & $83051112+/-$ \\
\hline $65061000-/+$ & $68070312-/+$ & $71052915-/+$ & $74060903+/-$ & $77040300+/-$ & $80072012+/-$ & $83052021+/-$ \\
\hline
\end{tabular}


Table 1. Continued.

\begin{tabular}{|c|c|c|c|c|c|}
\hline $83060800+/-$ & $86120412+/-$ & $90112000-/+$ & $94051400-/+$ & $98040300-/+$ & $01051800-/+$ \\
\hline $83061709+/-$ & $86122200-/+$ & $90120315-/+$ & $94052812+/-$ & $98040800+/-$ & $01060200+/-$ \\
\hline $83062600-/+$ & $87050906+/-$ & $90121700+/-$ & $94061009-/+$ & $98042321+/-$ & $01061500-/+$ \\
\hline $83070600+/-$ & $87052200+/-$ & $91010402+/-$ & $94062521+/-$ & $98051518-/+$ & $01062900+/-$ \\
\hline $83071109-/+$ & $87061000-/+$ & $91011206+/-$ & $94071312-/+$ & $98052009+/-$ & $01071200-/+$ \\
\hline $83072312-/+$ & $87061600+/-$ & $91012503-/+$ & $94072312+/-$ & $98052806-/+$ & $01072400+/-$ \\
\hline $83080200+/-$ & $87070800-/+$ & $91013100+/-$ & $94080900-/+$ & $98060309+/-$ & $01082100+/-$ \\
\hline $83080712-/+$ & $87071400+/-$ & $91020800+/-$ & $94082212+/-$ & $98061100-/+$ & $01111518-/+$ \\
\hline $83081200+/$ - & $87072600-/+$ & $91021912-/+$ & $94090500-/+$ & $98061806-/+$ & $01112406+/-$ \\
\hline $83081912-/+$ & $87080300-/+$ & $91022700+/-$ & $94091412+/-$ & $98070115+/-$ & $01121500-/+$ \\
\hline $83083000+/-$ & 87081112+/- & $91032112-/+$ & $94092515-/+$ & $98070912-/+$ & $01122400+/-$ \\
\hline $83090812+/-$ & $87083000-/+$ & $91041012-/+$ & $94101300+/-$ & $98071600+/-$ & $02010615-/+$ \\
\hline $83091512-/+$ & $87100712+/-$ & $91042412+/-$ & $94102300-/+$ & $98072103-/+$ & $02011906+/-$ \\
\hline $83092418+/-$ & $87102300-/+$ & $91050706-/+$ & $94110915+/-$ & $98091200-/+$ & $\odot 2 \odot 2050 \odot-/+$ \\
\hline $83100115-/+$ & $87110300+/-$ & $91052206+/-$ & $94111900-/+$ & $98100700-/+$ & $\odot 202170 \odot+/-$ \\
\hline $83101300-/+$ & $87111900-/+$ & $91061712+/-$ & $94120603+/-$ & $98101400+/-$ & $02030321-/+$ \\
\hline $83102012+/-$ & $87112900+/-$ & $91063000-/+$ & $94121500-/+$ & $98102712+/-$ & $02031800+/-$ \\
\hline $83102900-/+$ & $87121500-/+$ & $91071300+/-$ & $94121918+/-$ & $98110200-/+$ & $\odot 2050608+/-$ \\
\hline $83110112+/-$ & $87123103+/-$ & $91080100-/+$ & $94122400-/+$ & $98112312+/-$ & $02051900-/+$ \\
\hline $83110800-/+$ & $88011200-/+$ & 91081112+/- & $95010206+/-$ & $98113012-/+$ & $02060200+/-$ \\
\hline 83111418+/ - & $88021000-/+$ & $91082712-/+$ & $95011006-/+$ & 98121115+/- & ๑2061600-/+ \\
\hline $83112400-/+$ & $88022100+/-$ & $91090815+/-$ & $95011606+/-$ & $98121806-/+$ & $02062818+/-$ \\
\hline $83112812+/-$ & $88030800-/+$ & $91092418-/+$ & $95012106-/+$ & $99010500+/-$ & $\odot 2071206-/+$ \\
\hline $83120500-/+$ & $88032500+/-$ & $91100415+/-$ & $95012900+/-$ & $99011315-/+$ & $02072412+/-$ \\
\hline $83121112-/+$ & $88040400-/+$ & $91102100-/+$ & $95020600-/+$ & $99020221+/-$ & ๑2080818-/+ \\
\hline $83122200-/+$ & $88042012+/-$ & $91112900+/-$ & $95021012+/-$ & $99021112-/+$ & \\
\hline $84010100-/+$ & $88050412-/+$ & $91121600-/+$ & $95021618-/+$ & $99022812+/-$ & \\
\hline $84011000+/-$ & $88051600+/-$ & 91122709+/- & $95022606+/-$ & $99031000-/+$ & \\
\hline $84011900-/+$ & $88061403+/-$ & $92011000-/+$ & $95030412-/+$ & $99032906+/-$ & \\
\hline $84012803-/+$ & $88062906+/-$ & $92012700+/-$ & $95030900+/-$ & $99040915-/+$ & \\
\hline $84020312+/-$ & $88071512-/+$ & $92020100-/+$ & $95031721-/+$ & $99042608+/-$ & \\
\hline $84021500-/+$ & $88072500+/-$ & $92021900+/-$ & $95032606+/-$ & $99050515-/+$ & \\
\hline $84030100+/$ - & $88081200-/+$ & $92032109+/-$ & $95033009-/+$ & $99051203+/-$ & \\
\hline $84031300-/+$ & $88082000+/-$ & $92040200-/+$ & $95040615+/-$ & $99051600-/+$ & \\
\hline $84032700+/-$ & $88091015-/+$ & $92041806+/-$ & $95041506-/+$ & $99060115-/+$ & \\
\hline $84041012-/+$ & $88091700+/-$ & $92042800-/+$ & $95042212+/-$ & $99060815+/-$ & \\
\hline $84042400+/-$ & $88100500-/+$ & $92051800+/-$ & $95042600-/+$ & $99061515-/+$ & \\
\hline $84050600-/+$ & $88101400+/-$ & $92052600-/+$ & $95050203+/-$ & $99071303-/+$ & \\
\hline $84051712+/-$ & $88110100-/+$ & $92062300-/+$ & $95052312-/+$ & $99073006+/-$ & \\
\hline $84060112-/+$ & $88111100+/-$ & $92070100+/-$ & $95053003+/-$ & $99080602-/+$ & \\
\hline $84061500+/-$ & $88112600-/+$ & $92071100-/+$ & $95060900-/+$ & $99081600-/+$ & \\
\hline $84062821-/+$ & $88121100+/-$ & $92072012-/+$ & $95061408+/-$ & $99082412+/-$ & \\
\hline $84071200+/-$ & $88122218-/+$ & $92072700+/-$ & $95061912-/+$ & $99090702+/-$ & \\
\hline $84072700-/+$ & $89010312+/-$ & $92080500-/+$ & $95062512+/-$ & $99091206-/+$ & \\
\hline $84080800+/-$ & $89012000-/+$ & $92082600-/+$ & $95071300-/+$ & $99092212+/-$ & \\
\hline $84082300-/+$ & $89013100+/-$ & $92092612-/+$ & $95072400+/-$ & $99092618-/+$ & \\
\hline $84090500+/-$ & $89021600-/+$ & $92101300+/-$ & $95080100-/+$ & $99100215+/-$ & \\
\hline $84091900-/+$ & $89030100+/-$ & $92102612-/+$ & $95081912+/-$ & $99101000-/+$ & \\
\hline $84100300+/-$ & $89031400-/+$ & $92110800+/-$ & $95082500-/+$ & $99102912+/-$ & \\
\hline $84101800-/+$ & $89032706+/-$ & $92112212-/+$ & $95091603+/-$ & $99110700-/+$ & \\
\hline $84102700+/-$ & $89041115-/+$ & $92120718+/-$ & $95100212-/+$ & $99120309-/+$ & \\
\hline $84111400-/+$ & $89042515+/-$ & $92121900-/+$ & $95103012-/+$ & 99121318+/- & \\
\hline $84112300+/-$ & $89051115-/+$ & $93010100+/-$ & $95112200+/-$ & $99122400-/+$ & \\
\hline $84121000-/+$ & $89052315+/-$ & $93012400-/+$ & $95112700-/+$ & $99123018-/+$ & \\
\hline $84122100+/-$ & $89062412+/-$ & $93013100+/-$ & $95122400-/+$ & $00011000+/-$ & \\
\hline $85010812-/+$ & $89070600-/+$ & $93030100+/-$ & $95123100+/-$ & $0 \odot 012000-/+$ & \\
\hline $85013100-/+$ & $89071500-/+$ & $93032006-/+$ & $96040815+/-$ & $00012712-/+$ & \\
\hline $85022700-/+$ & $89072300+/-$ & $93050609+/-$ & $96042600-/+$ & $00020518+/-$ & \\
\hline $85032800-/+$ & $89081000-/+$ & $93051300-/+$ & $96060412-/+$ & $00030600+/-$ & \\
\hline $85050400+/-$ & $89101600-/+$ & $93060300+/-$ & $96061509+/-$ & $00031600-/+$ & \\
\hline $85052015-/+$ & $89102906+/-$ & $93061006-/+$ & $96061902-/+$ & $00041606-/+$ & \\
\hline $85053112+/-$ & $89112100+/-$ & $93062312-/+$ & $96070112-/+$ & $00042712+/-$ & \\
\hline $85061700-/+$ & $89121809+/-$ & $93062900+/-$ & $96071200+/-$ & $00051212-/+$ & \\
\hline $85062503+/-$ & $90010800-/+$ & $93070800-/+$ & $96080700+/-$ & $00052400+/-$ & \\
\hline $85071200-/+$ & $90011609+/-$ & $93072700+/-$ & $96090412+/-$ & $00062200+/-$ & \\
\hline $85072300+/-$ & $90020415-/+$ & $93080500-/+$ & $96091000-/+$ & $00070412-/+$ & \\
\hline $85081000-/+$ & $90021600+/-$ & $93082300+/-$ & $96092900+/-$ & $00073121-/+$ & \\
\hline 85081800+/ - & $90030318-/+$ & $93090200-/+$ & $96100800-/+$ & $00082718-/+$ & \\
\hline $85090600-/+$ & $90031400+/-$ & $93092812-/+$ & $96102709+/-$ & $00092418-/+$ & \\
\hline $85091406+/-$ & $9 \odot \odot 4 \odot 212-/+$ & $93100512+/-$ & $96110402-/+$ & $\odot \odot 102206-/+$ & \\
\hline $85100300-/+$ & $90041000+/-$ & $93102100+/-$ & $96112406+/-$ & $00110403-/+$ & \\
\hline $85101109+/-$ & $90050100-/+$ & $93110318-/+$ & $96120912-/+$ & $00111815-/+$ & \\
\hline $85103112-/+$ & $90051006+/-$ & $93120109-/+$ & $96122106+/-$ & $00112318+/-$ & \\
\hline $85110900+/-$ & $90060600+/-$ & $93121509+/-$ & $97010700-/+$ & $00120700+/-$ & \\
\hline $85112700-/+$ & $90062300-/+$ & $93123000-/+$ & $97030512-/+$ & $00121700-/+$ & \\
\hline $85120700+/-$ & $90062906+/-$ & $94011106+/-$ & $97031203+/-$ & $00122300+/-$ & \\
\hline $85122600-/+$ & $90071900-/+$ & $94012600-/+$ & $97041612+/-$ & $01010412+/-$ & \\
\hline $86010612+/-$ & $90072512+/-$ & $94020612+/-$ & $97070306-/+$ & $01011912+/-$ & \\
\hline $86012200-/+$ & $90081300-/+$ & $94022200-/+$ & $97090806+/-$ & $01012815-/+$ & \\
\hline $86021800-/+$ & $90091106-/+$ & $94030500+/-$ & $97100703-/+$ & $01021300+/-$ & \\
\hline $86053100+/-$ & $90092500+/-$ & $94032100-/+$ & $97111612+/-$ & $01022700-/+$ & \\
\hline $86062709+/-$ & $90100912-/+$ & $94040200+/-$ & $97123003-/+$ & $01031212+/-$ & \\
\hline $86072421+/-$ & $90102200+/-$ & $94041800-/+$ & $98010600+/-$ & $01042309-/+$ & \\
\hline $86112500-/+$ & $90110712-/+$ & $94050100+/-$ & $98031009+/-$ & $01050609+/-$ & \\
\hline
\end{tabular}



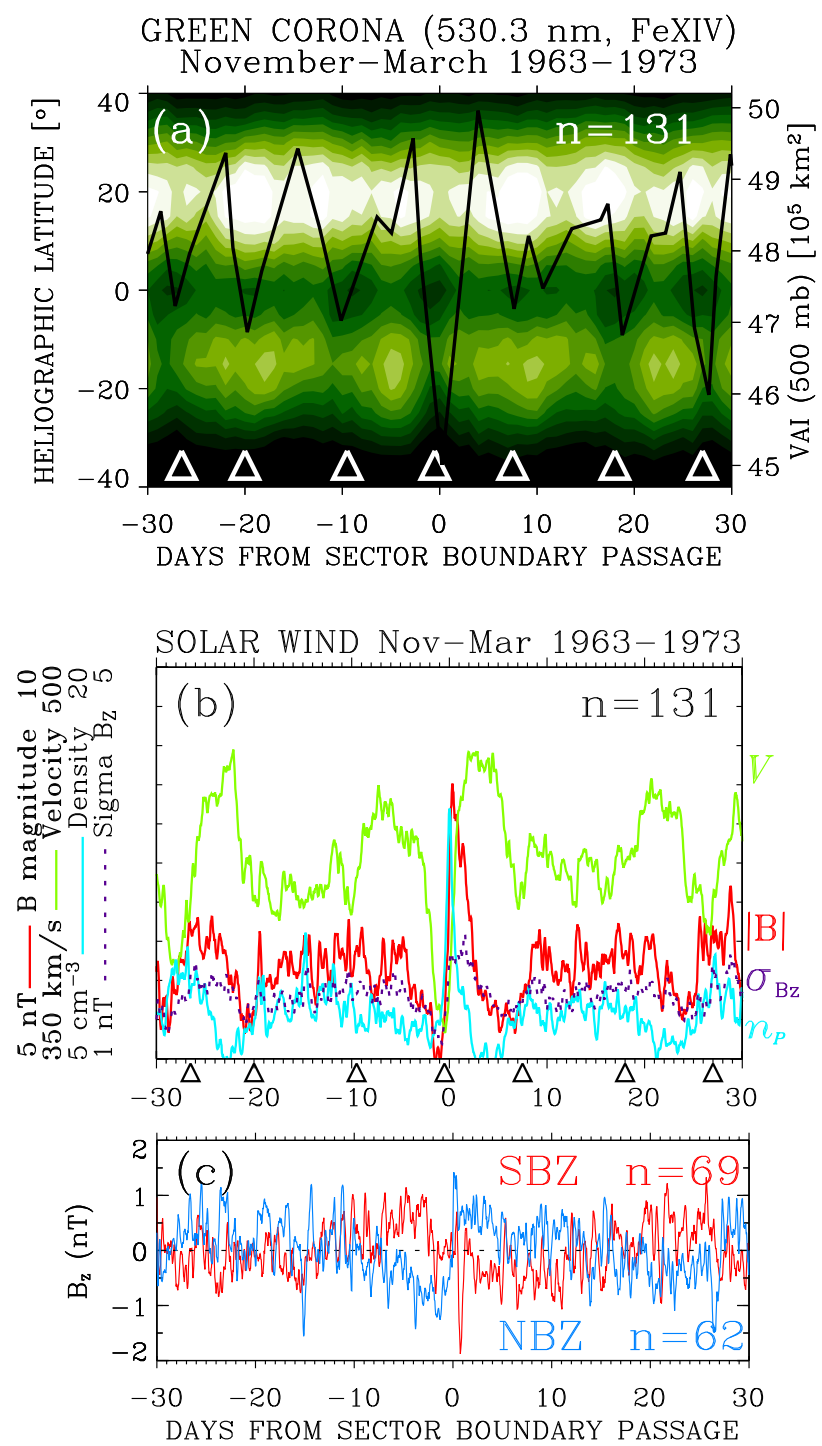

Fig. 3. The superposed epoch analysis of (a) the green solar corona emission line intensity interpolated for the central meridian and (bc) solar wind plasma parameters. All 131 60-day long time series are keyed by the magnetic sector boundary passage that occurred during the northern "winter" of November-March from 1963 to 1973. Triangles indicate times of mean coronal holes passing the solar central meridian. The VAI result by Wilcox et al. (1976, their Fig. 4) is superposed in the top panel.

Knowing the polarity reversals of the magnetic sectors for SBP events (Table 1) we can approximate the rule applied by Miyoshi and Kataoka (2008) and divide the dataset of SBP events into group A (SBZ events) and group B (NBZ events). The IMF $B_{Z}$ for these two groups of events shows mean trends that are similar to those found by Miyoshi and Kataoka (2008) for CIRs, except for sector boundaries the IMF $B_{Z}$ does not merely shift from near zero values but there is a reversal of $B_{Z}$. It should be noted that the IMF polarity given in Table 1 is toward (-) and away (+) from the Sun. Miyoshi and Kataoka (2008) use the IMF azimuthal angle sectors to define the predominant direction of the magnetic field after the stream interface with the same sense of away/toward polarity. For the NH cold season, we select SBP events of the type SBZ if they occur either in January-March when the SBP polarity is $+/-$ or November-December when the SBP polarity is $-/+$. Conversely, we select events of the type NBZ for the same months but when the corresponding SBP polarities are opposite. For the SH cold season, we select SBP events of the type SBZ if they occur either in May-June when the SBP polarity is $+/-$ or July-September when the SBP polarity is $-/+$. The NBZ events are from the same months but when the corresponding SBP polarities are opposite. As discussed by Miyoshi and Kataoka (2008), according to the Russell-McPherron effect, geomagnetic activity is enhanced (due to a tendency of $B_{Z}$ to be southward) when so-called "spring-toward fall-away" rule applies, because the polarities of toward and away have a larger projection component of the southward IMF around spring and fall equinoxes, respectively. This rule defines the type SBZ events and the viceversa rule defines the NBZ events. In other words, we use the same rules except that we include months of January and June to extend the "spring months" period of February-May. Similarly, July and December are included to extend the "fall months" period of August-November used by Miyoshi and Kataoka (2008). When the SBP events are classified as type $\mathrm{SBZ}$ or NBZ, the corresponding mean trends of the IMF $B_{Z}$ trends are anticorrelated (Fig. 3c). For SBZ events the mean IMF $B_{Z}$ reverses from northward to southward, while it reverses from southward to northward for NBZ events. This is considered below in the discussion of VAI results.

The approximate phase alignment between the solar wind structure and the main VAI minimum observed by Wilcox et al. led Hines and Halevy (1977) to a conclusion regarding a possible interpretation of the Wilcox effect. They suggested that an unknown operative solar mechanism modulates the phase of tropospheric processes. It should be noted that these arguments were based solely on the results for the interval of \pm 6 epoch days, the VAI minimum in particular. As far as we know, the VAI variations for epoch days beyond this period were considered only to show that their amplitudes were smaller than the central pattern, which was thus found statistically significant (Wilcox et al., 1976). The sector boundary and CIR are associated with the onset of solar wind fluctuations and can serve as approximate markers of the onset of intense auroral activity and ensuing atmospheric gravity waves, which are invoked as a possible mechanism (Prikryl et al., 2009) to explain the observed solar wind - troposphere link.

The reality of the Wilcox effect has been questioned and several studies have attempted to confirm or disprove it (see Sect. 1). Hines and Halevy (1977) showed high statistical significance of these results and Kirkland et al. (1996) pointed out possible environmental conditions that may have 
resulted in disappearance of the effect. With the present availability of an extensive solar wind database (OMNI) and recent re-analysis projects (e.g. ECMWF ERA-40) it is now possible to re-visit the analysis and verify the effect for an extended period in both the Northern and Southern Hemispheres. Time series of 12-hourly relative vorticity for 500and 300-mb levels extracted from the ERA-40 database and converted to absolute vorticity (see, e.g. Holton, 1992) for latitude band between $20^{\circ}$ and $80^{\circ}$ for both hemispheres are used to compute VAI as defined by Roberts and Olson (1973) and used by Wilcox et al. (1974). Briefly, VAI is the total area where the absolute vorticity is above a given threshold. Although a single threshold value can be used these authors employed two threshold values $\left(20 \times 10^{-5} \mathrm{~s}^{-1}\right.$ and $24 \times 10^{-5} \mathrm{~s}^{-1}$ ) to compute VAI for any given time by summing up two areas in $\mathrm{km}^{2}$ where the absolute vorticity exceeded these thresholds. The index seems to characterize the level of storminess in a given geographical sector. The same thresholds are used in the present analysis, which is only concerned with the relative variation of the index.

For the northern and southern latitude bands of $20^{\circ}-80^{\circ}$, the SPE analysis of VAI at the 500-mb level is performed using 131 and 143 SBP events that occurred during "winter" months of November-March and May-September 19631973, respectively (Fig. 4a). The "winter" is defined in accordance with the month selection by Wilcox et al. $(1975,1976)$. For the northern and southern winters, very similar and statistically significant mean VAI variations are found. To check the persistence or reproducibility of the Wilcox effect, we have initially split the data into two sub-periods 1963-1968 and 1969-1973, for which comparable results are obtained (not shown). Following the suggestion of a reviewer we then considered 7 years of high aerosol concentration after the eruption of Agung volcano (1964-1970) and 4 years of low (1963 and 1971-1973) stratospheric aerosol. The SPE results are shown (Fig. 4a) by red/dotted and green/dashed lines for high and low aerosol cases, respectively. The Wilcox effect is found in both cases with similar amplitudes in the Northern and Southern Hemispheres. For the Northern Hemisphere, the overall mean VAI variations agree well with the result by Wilcox et al. (1976, their Fig. 4) that is superposed in Fig. 4a (heavy broken line).

Figure $4 \mathrm{~b}-\mathrm{c}$ shows the corresponding mean solar wind parameters (the IMF $B_{Z}$ in Fig. $4 \mathrm{c}$ is discussed below). As already shown for the northern winter (Fig. 3b), the solar wind data show superposed streams and CIRs, the key CIR at day 0 being the sharpest. There is one-to-one correspondence between the mean stream interfaces (CIRs) and VAI minima with a delay of about 1 day as shown (for the Southern Hemisphere winter) by triangles in Fig. $4 \mathrm{a}$ and $\mathrm{b}$. The mean stream interfaces near the minima of the mean solar wind speed are followed by solar wind speed maxima 1-3 days later. Thus the mean solar wind speed maxima are followed by mean VAI maxima with a similar delay.
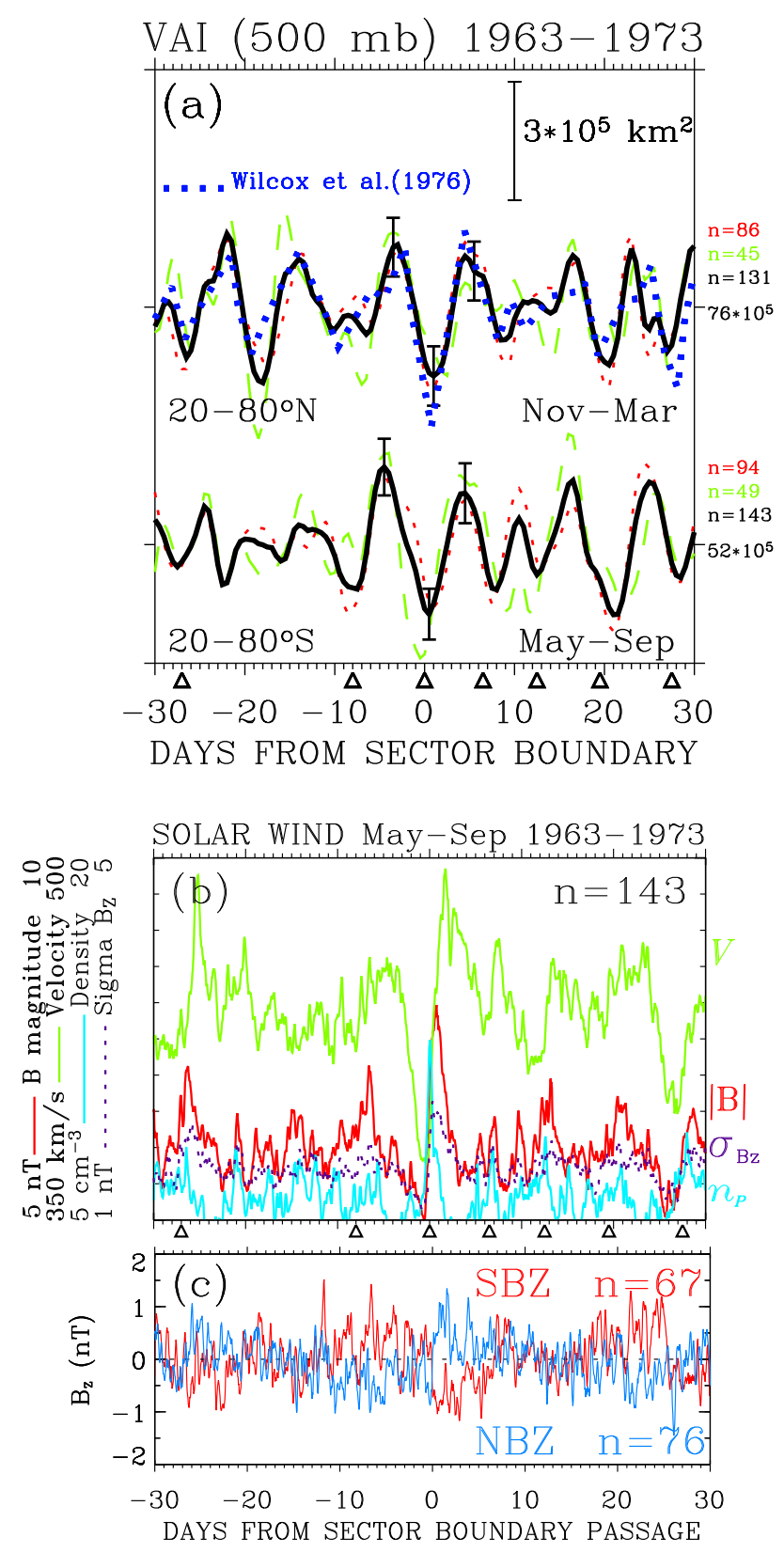

Fig. 4. The superposed epoch analysis of (a) VAI for 500-mb level and (b-c) solar wind plasma parameters. The VAI time series are keyed by the magnetic sector boundary passage that occurred during the northern and southern winters 1963-1973. The results for VAI are shown for subintervals of high and low volcanic aerosol loading by dotted red and broken green lines, respectively. The overall mean over the 60 day period is shown by the black line. The solar wind results are shown for the Southern Hemisphere winter. Triangles indicate approximate times of mean stream interfaces.

For the 60-day SPE data in Fig. 4, most of the mean solar wind speed maxima are associated with depletions of the mean coronal intensity (not shown). The streams and associated CIRs were spaced more irregularly and spread over the 
epoch days between day -25 and day -10 thus lacking coherence when superposed. This is matched by low amplitude VAI variations for the same interval. However, a well defined mean stream interface (CIR) near day -27 is associated with a mean coronal hole and there is a corresponding bipolar VAI signature (minimum/maximum) near day -27 that is associated with the sharp solar wind enhancement though small in amplitude. Similar recurrent patterns in the solar wind, solar corona and VAI (weaker versions of the central patterns near day 0) are repeated two solar rotations later near day +28 .

While the inter-hemispheric correlation between the mean VAI variations is low for the negative epoch days less than -10 days, it is remarkably high later on. There is one-toone correspondence between VAI maxima/minima with uncertainty of \pm 2 days, which is clearly a result of very similar mean solar wind structure during the northern and southern winters as shown in Figs. $3 \mathrm{~b}$ and $4 \mathrm{~b}$, respectively. A one-toone relationship between mean solar wind streams and VAI for both northern and southern winters suggests a modulation of VAI by solar wind streams. The mean VAI variations at 300-mb level (not shown for this period) have greater amplitudes and are in phase with those for $500 \mathrm{mb}$. In Sect. 4.2 we extend the VAI for 500 and 300-mb levels to years 19632002, for which both solar wind and ERA-40 re-analysis data are available.

Now we discuss the mean IMF $B_{Z}$ component that fluctuates between small positive and negative values if averaged for all SBP events (not shown in Fig. 4c). However, splitting the SBP events into groups A (SBZ events) and B (NBZ events) as discussed above we obtain anticorrelated trends that are similar to those shown in Fig. 3c. The SPE analysis of other solar wind plasma parameters including the IMF magnitude and $\sigma_{B z}$, which is largely due to solar wind Alfvén waves, results in the same amplitudes for SBZ and NBZ events (Miyoshi and Kataoka, 2008, their Fig. 1). When the VAI data is grouped by the polarity of $B_{Z}$, the Wilcox effect is well reproduced in both cases and in both hemispheres (not shown). The impact of the IMF $B_{Z}$ orientation on the phase and amplitude of the Wilcox effect is discussed in Sect. 4.2.

\subsection{The VAI results for extended period (1963-2002)}

Figure 5a and $\mathrm{b}$ shows the results of the SPE analysis of VAI at 500 and $300 \mathrm{mb}$. The VAI time series are keyed by 432 and 438 SBP events that occurred during the Northern and Southern Hemisphere winters, i.e. November-March and MaySeptember, respectively. The overall central bipolar VAI pattern of a minimum near the key day followed by a maximum a few days later is found for both levels in both hemispheres. The persistence or reproducibility of the effect was initially checked by splitting the data into two sub-periods 1963-1982 and 1983-2002 (not shown). We then subdivided the data into periods of high (1964-1970, 1983-1986 and 1992-1996; shown in red lines) and low (1963, 1971-1982,
1987-1991 and 1997-2002; shown in green lines) volcanic aerosol loading. With exception of the Northern Hemisphere VAI at $500 \mathrm{mb}$, the Wilcox effect persists through both high and low aerosol periods with similar mean VAI amplitudes.

The VAI mean variations for the 500-mb level are in phase with those at $300 \mathrm{mb}$ though the former have smaller amplitudes. Consistent with the previous results (Tinsley et al., 1994; Kirkland et al., 1996) for the Wilcox effect, the VAI minimum near the epoch day +1 is weak at $500 \mathrm{mb}$ during years of low aerosol loading. However, near day +26 (approximately one solar rotation later) a characteristic bipolar signature of VAI, i.e. a minimum followed by a maximum, is found that is persistent during years of low and high aerosol loading. In the Southern Hemisphere, the effect near day +1 is very well reproduced through sub-periods of low and high aerosols when the data set is split into high and low aerosol periods. The lack of persistence of the effect or a missing minimum near day +1 at $500 \mathrm{mb}$ for the Northern Hemisphere during years of low aerosol loading was interpreted by Tinsley et al. as supporting of the AEC mechanism, which requires the presence of aerosols for a significant Wilcox effect. The latter interpretation is clearly not supported in the Southern Hemisphere. Also, the Northern Hemisphere 500-mb results (Fig. 5a) for low aerosol years, while not showing a minimum just after day 0 , still show a well reproduced trend of the VAI enhancement after the key day and a good overall correlation between the "aerosol" and "no aerosol" cases. Furthermore, the effect persists at $300 \mathrm{mb}$ in both hemispheres regardless of the aerosol loading.

Because the separation of coronal holes and corresponding high-speed streams varies between $\sim 5$ and 27 days, it is expected that any modulation of VAI by solar wind streams that preceded or followed the key stream becomes less evident when the SPE analysis spans 40 years. The out-ofphase variations are partly cancelled out and generally not reproduced for the two subintervals. However, typical separations of coronal holes and solar wind streams are $\sim 7,9$ and 14 days, i.e. approximately integral fractions of the synodic solar rotation so that the modulation of VAI for epoch days other than day 0 may still be partly reinforced and sometimes reproduced for both sub-intervals.

Figure 5c shows a one-to-one correspondence between mean coronal holes (indicated by triangles) and the $300-\mathrm{mb}$ VAI minima for the Southern Hemisphere winters with a delay of about 1-2 days. This correlation is partly destroyed for the mean coronal hole near day +16 where the VAI variations for two sub-periods are generally out of phase (Fig. 5b). The corresponding solar wind data are shown in Fig. 5d. The strongest composite CIRs and mean streams are associated with the largest mean coronal holes recurring with period of about 27 days. The mean CIRs and streams associated with the weak mean coronal holes, particularly for epoch days -20 and +8 are smeared out.

The importance of the IMF $B_{Z}$ for the Wilcox effect is further examined in Figs. 5e-f and 6a-b. The SPE analysis of 

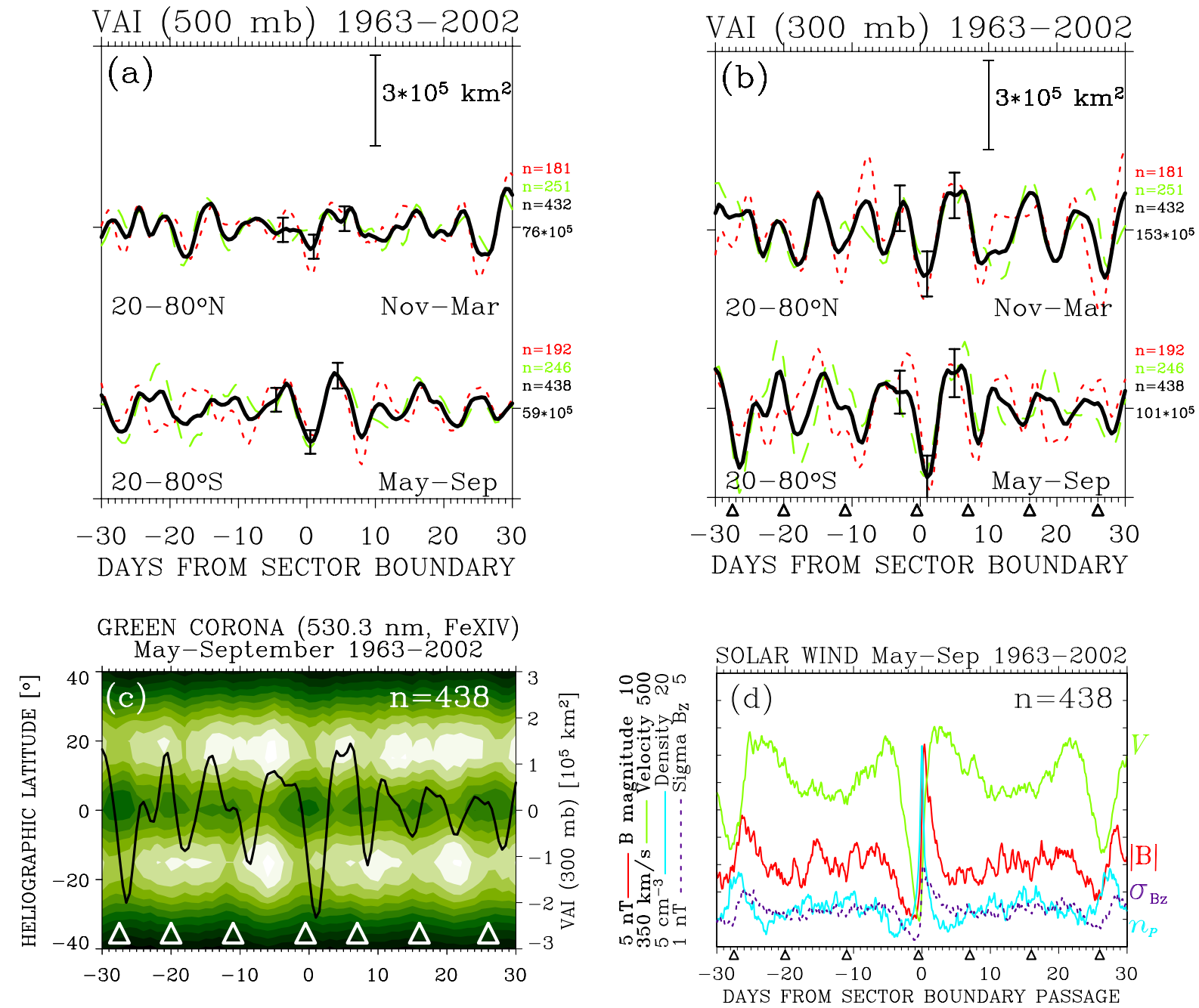

Fig. 5. The superposed epoch analysis of VAI at (a) 500-mb and (b) 300-mb levels, (c) the green solar corona emission line intensity interpolated for the central meridian and (d) solar wind plasma parameters. The time series are keyed by the magnetic sector boundary passage. The VAI results for the northern and southern winters 1963-2002 are subdivided into periods of high and low volcanic aerosol loading shown by dotted red and broken green lines, respectively. The solar corona and solar wind data for the southern winters are shown. Triangles indicate approximate mean times of coronal holes.

the IMF $B_{Z}$ for all SBP events gives no trend and the mean $B_{Z}$ fluctuates about zero between small positive and negative values (not shown). When the SBP events are classified as SBZ and NBZ, as discussed above, the mean $B_{Z}$ switches sign on day 0 (Fig. $5 \mathrm{~g}-\mathrm{h}$ ). This is a much steeper change than in Figs. $3 \mathrm{c}$ and $4 \mathrm{c}$ because more and higher quality data are added. Also, it is a reversal of $B_{Z}$ comparing to a shift from near zero values for CIRs (Miyoshi and Kataoka, 2008, their Fig. 1). In general, SBPs do not coincide with CIRs but there is always (by definition of sector boundary) a discontinuity in the IMF components, $B_{X}$ in particular, associated with SBPs. When the SPE analysis of VAI at $300 \mathrm{mb}$ is done separately for the SBZ and NBZ events, the Wilcox effect is well reproduced with large amplitudes in both cases and in both hemispheres (not shown separately for $\mathrm{NH}$ and $\mathrm{SH}$ ). Figure $5 \mathrm{e}$ and $\mathrm{f}$ shows the results for VAI at $500 \mathrm{mb}$, which gives weaker response in the Northern Hemisphere when all SBP events are considered (Fig. 5a). For SBZ events (Fig. 5e), there are well defined VAI maxima at about 3-4 days after SBP (day 0) that persist during high- and low-aerosol years in both hemispheres. However, in the Northern Hemisphere for SBZ events, the VAI minimum (Wilcox effect) is broadened into negative epoch days that are associated with northward $B_{Z}$ before it switches to southward. For NBZ events (Fig. 5f), the maxima are again reproduced in both hemispheres but are delayed to about 5-6 days after the key day. The Wilcox effect (minimum) is well reproduced in the Southern Hemisphere. In the Northern Hemisphere, a strong 

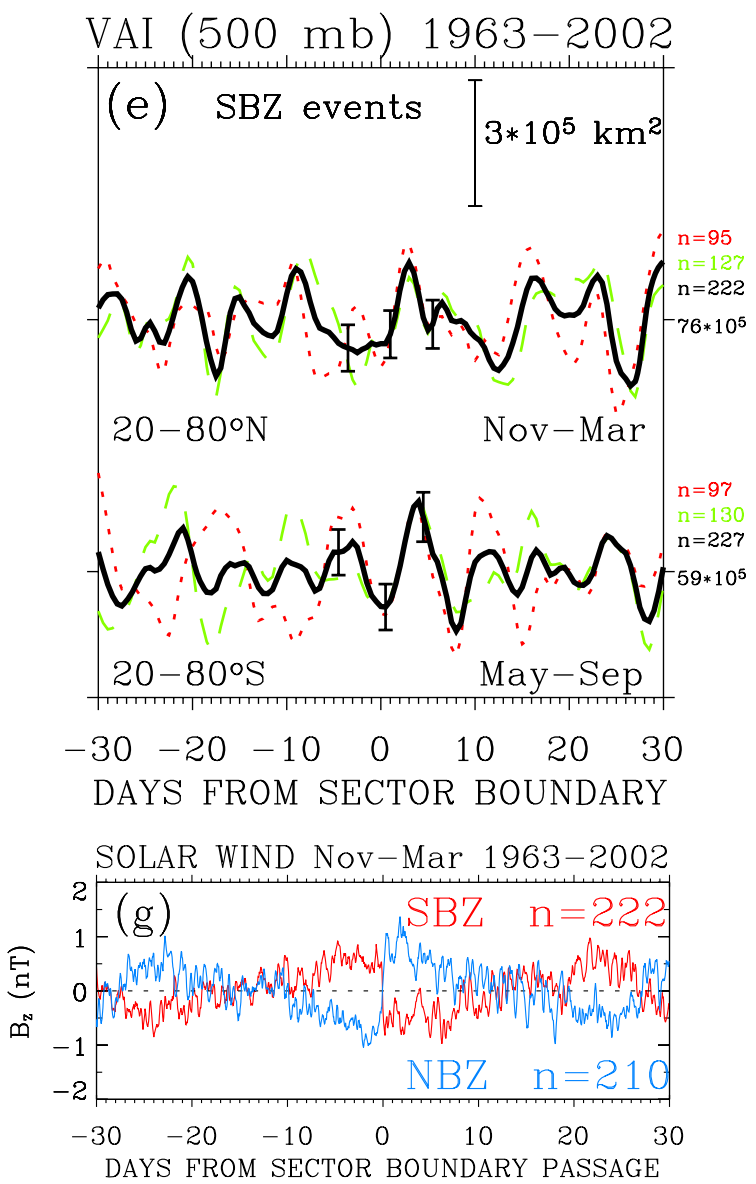
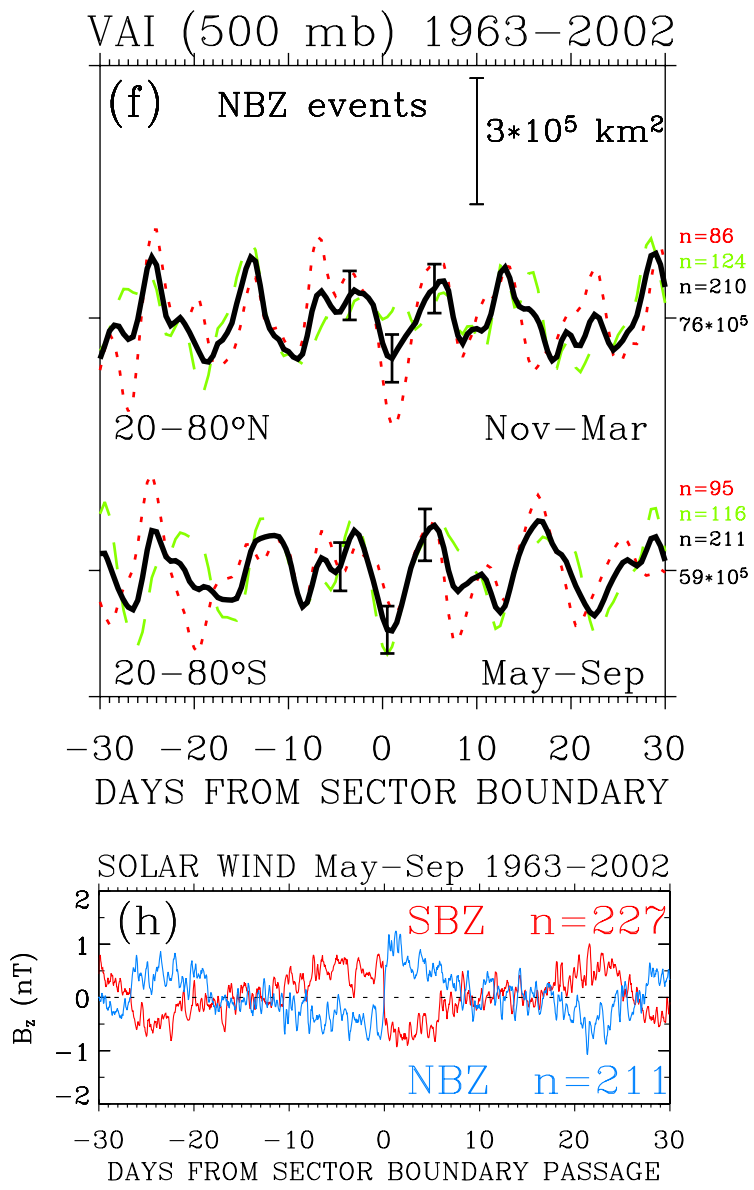

Fig. 5. Continued. The superposed epoch analysis of (e-f) VAI at 500-mb level, and (g-h) IMF $B_{Z}$ component. The time series are keyed by the magnetic sector boundary passage belonging to group A (SBZ) and group B (NBZ). The VAI results for the northern and southern winters 1963-2002 are further subdivided into periods of high and low volcanic aerosol loading shown by dotted red and broken green lines, respectively.

Wilcox effect is found during high-aerosol years but no minimum near day +1 is found during low-aerosol years for NBZ events (Fig. 5f). However, coherent (in-phase) variations of VAI with periods 9-10 days are found at other epoch days, both in the NH and SH. In particular, it should be noted that peaks at -25 and +29 days are separated by two synodic solar rotations. These VAI peaks are associated with reversals of the IMF $B_{Z}$ that are more gradual than the reversal at day 0 (Fig. 5g-h).

The influence of the IMF $B_{Z}$ and/or the lack of the influence of aerosols on Wilcox effect at 500- and 300-mb levels is summarized in Fig. $6 \mathrm{a}-\mathrm{b}$ by combining the $\mathrm{NH}$ and $\mathrm{SH}$ data for the NBZ and SBZ events. In both cases, the VAI variations around the key day persist during years of high and low volcanic aerosols, including the 500-mb VAI minimum near day +1 for low-aerosol years. The match in phase and amplitude of the 500-mb VAI variations (Fig. 6a) for high and low aerosol years is particularly close for NBZ events.
Even though the key minimum is weak or missing during low-aerosol years in the NH (Fig. 5e-f), the combined results suggest there is no aerosol dependence of the effect.

Although the IMF $B_{Z}$ does not seem to significantly affect the overall amplitude of the Wilcox effect, the VAI minimum is more prominent for NBZ events and there is a phase shift. At least at $300 \mathrm{mb}$ (Fig. 6b), the key VAI minimum is shifted by one day from +0.5 to +1.5 days for SBZ and NBZ events, respectively. A smaller phase shift is found for the VAI minimum at $500 \mathrm{mb}$. The VAI maximum that follows occurs between days +3 and +4 for SBZ events, while it is shifted to day +5.5 for NBZ events, both at 300 and $500 \mathrm{mb}$. This is consistent with higher and lower geomagnetic activity following SBZ and NBZ events, respectively (Miyoshi and Kataoka, 2008). If auroral AGWs play a role in stimulating the growth of extratropical cyclones (Prikryl et al., 2009) they should be more effective during SBZ events, which indeed suggest a faster growth to the VAI peak than is the case 
for NBZ events. Also, there are significantly higher VAI values prior to day 0 (VAI maxima between day -3 and -8 ) for NBZ events in comparison with SBZ events. The NBZ events are associated with a period of southward $B_{Z}$ before the reversal to northward at day 0 and vice versa for SBZ events, which is consistent with the same argument invoking more intense auroral AGWs when $B_{Z}$ is southward $(<0)$. Finally, when $B_{Z}$ turns northward (NBZ events) the intensity of solar wind coupling and AGWs weakens thus leading to a deeper VAI minimum followed by a delayed VAI maximum. Conversely, when $B_{Z}$ turns southward (SBZ events) the intensity of solar wind coupling and AGWs is greater thus leading to a shallower VAI minimum followed by an advancing (faster growing) VAI maximum.

Based on the Northern Hemisphere 500-mb vorticity data for \pm 6 days from the heliospheric currents sheet (HCS) transit, Tinsley et al. (1994) and Kirkland et al. (1996) have suggested that the AEC mechanism requires the presence of stratospheric aerosols for a significant Wilcox effect. These authors used the HCS transit, which is interchangeable with the SBP term. Using the ECMWF ERA-40 re-analysis of the 300- and 500-mb vorticity data, which is of higher quality than the NMC gridded data set used by the above authors, we find that the Wilcox effect persists through periods of low as well as high aerosol loading, with the exception of the Northern Hemisphere at $500 \mathrm{mb}$ during low-aerosol years after 1973. However, as shown above, the VAI variations for this case are coherent and well reproduced at other epoch times. Furthermore, when the data are stratified by $B_{Z}$ (SBZ or NBZ events) and combined for the $\mathrm{NH}$ and $\mathrm{SH}$, no dependence on aerosols is found. The phase shift between the VAI responses obtained for these two subsets may explain the reduced amplitude of the overall Wilcox effect.

If the Wilcox effect did not persist through low aerosol levels, that would be a strong argument against the mechanism involving AGWs, while it would support the AEC mechanism, particularly if confirmed in the Southern Hemisphere and at 300-mb level. However, our results, while in agreement with those obtained by Tinsley et al. (1994) and Kirkland et al. (1996) for the 500-mb level in the Northern Hemisphere, do not support their interpretation of the Wilcox effect based on the requirement of the aerosol presence for the AEC mechanism. This does not necessarily eliminate the possibility that the AEC affects cloud microphysics, as the reduced aerosol loading may not entirely remove the effect (Tinsley and Zhou, 2006). Kniveton and Tinsley (2004) used global cloud products from ISCCP-D1 dataset to examine the changes in cloud cover following Earth transits by the HCS for periods of high and low stratospheric aerosol loading. These authors tentatively concluded that their results for events when stratospheric aerosol loading is high and the relativistic electron flux (REF) declines, during the period of low solar wind velocity just prior to the arrival of the HCS, concur with the expected behaviour due to AEC mechanism. However, they raise the possibility that the changes in cloud
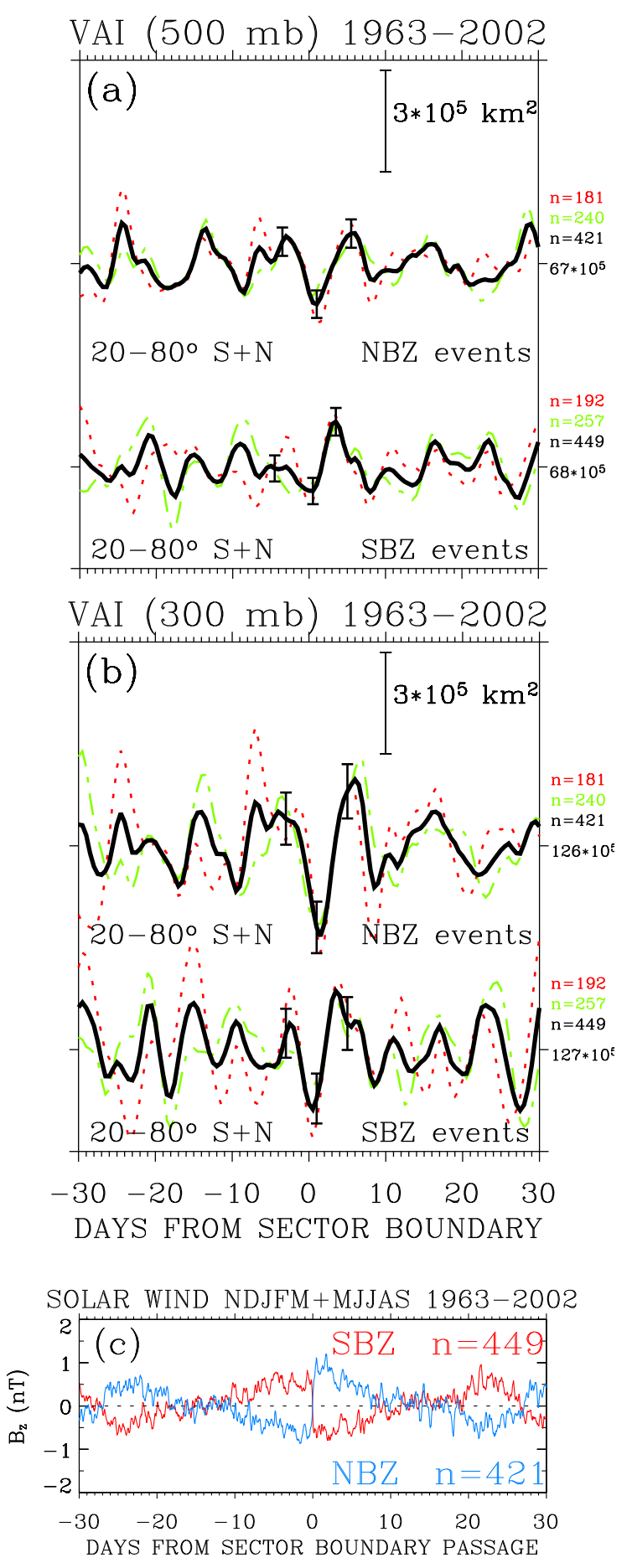

Fig. 6. The superposed epoch analysis of VAI at (a) 500-mb and (b) 300-mb levels combining the Southern and Northern Hemisphere data, and (c) IMF $B_{Z}$ component for SBZ and NBZ events. The time series are keyed by the magnetic sector boundary passage. The VAI results for 1963-2002 are further subdivided into periods of high and low volcanic aerosol loading shown by dotted red and broken green lines, respectively. 
cover are not purely related to changes in the REF as the Earth transits the HCS.

The IMF sector boundaries have been regarded merely as markers of some unknown process associated with the solar wind structure that causes the observed reduction in VAI (Wilcox et al., 1974). The reduction in solar wind velocity on average 1-2 days prior the SBP transit (Fig. 2b) is not only associated with the observed reduction in precipitating REF, but also a calm solar wind period characterized by weak $B$ and absence of Alfvén waves. This is typical of periods that precede enhanced geomagnetic (storm) activity (Borovsky and Steinberg, 2006) caused by the subsequent high-speed plasma stream arrival marked by the CIR. On average, the IMF sector boundaries closely precede the CIRs (stream interfaces) as demonstrated in Fig. 2b. The AEC mechanism in conjunction with reduced REF and high atmospheric aerosol loading aims to explain the observed VAI minimum but does not address the VAI maxima, which also were not discussed by Wilcox et al. and in subsequent studies of VAI correlations with solar wind. The present results that are supported by coronal intensities suggest that there is a component of VAI variation that is in phase with waxing and waning solar wind streams. Thus there is a need to identify a mechanism that can explain not only the VAI reduction but also the VAI enhancements. The AGW mechanism proposed in this paper and the companion paper links the calm solar wind, the absence or reduced amplitude of Alfvén waves in particular, with the VAI minimum. The VAI maxima that follow and precede this minimum (Figs. 4a, 5a-b, 5e-f and 6a-b) are associated with the high-speed solar wind (Figs. 4b and 5d), which is rich in large amplitude solar wind Alfvén waves, a source of AGWs. Furthermore, as discussed above, the IMF $B_{Z}$ seems to control the phase of the VAI pattern as well as the depth of the VAI minimum. In principle, the AEC and AGW mechanisms may work in synergy but the latter also points to a possibility of solar wind influence on the growth of extratropical cyclones (Sect. 5).

\subsection{The spectra of solar wind/corona and VAI time se- ries}

As already discussed (Sect. 4.1), the observed tendency of phase alignment between the solar wind forcing and VAI is consistent with the conclusion by Hines and Halevy (1977) that the Wilcox effect is likely a phase modulation of tropospheric processes. Typically, the key VAI minimum is preceded and followed by maxima, which are approximately 69 days apart and are common time separations between two successive solar wind streams. The mean solar wind speed observed by spacecraft near the Earth shows similar temporal patterns with a lead time of $\sim 1-2$ days. Of course, the time separation of successive streams is determined by heliospheric longitude separation of coronal holes, which is commonly $360^{\circ} / n$, where $n=1,2,3$ or 4 . There may be $1-4$ recurrent coronal holes per solar rotation (27.3 days) and thus typical time separations of major recurrent plasma streams are $\sim 27,13.5,9$ and 7 days. The SPE analysis picks out the average separation. During solar cycle maxima, CMEs generally disrupt the recurrent solar wind pattern but they can also be a source of auroral AGWs that may impact the development of extratropical cyclones.

To show the relationship between the solar wind and VAI in the frequency domain we have computed Fast Fourier Transform (FFT) spectra of year-long time series of daily means of the coronal intensity between $-10^{\circ}$ and $+10^{\circ}$ of heliographic latitude, solar wind velocity, density and magnetic field magnitude, and VAI at 300 and $500 \mathrm{mb}$. To remove the DC and low frequency components a 50-day running average was subtracted. The Hanning filter was applied before the FFT. While the position of peaks in the FFT spectra varies from year to year, the spectra averaged over 40 years (1963-2002) show peaks near the characteristic periods discussed above. It should be noted that solar wind time series were interpolated for data gaps. However, this did not significantly affect the positions of the average spectral peaks, although the peaks are sharper after 1995 when there were continuous solar wind data. Figure 7 a shows the normalized spectra for the green corona and solar wind parameters for the interval 1995-2005. Figure 7b-c shows the spectra of the green corona intensity, the IMF magnitude, and VAI at 300 and $500 \mathrm{mb}$ for the interval from 1963 to 2002. The VAI spectra for the Northern Hemisphere are computed for time series centered at 1 January so that the Hanning filter does not reduce amplitudes in winter months. Apart from the peak near the synodic solar rotation period the VAI spectral peaks are not as pronounced as the peaks for the corona and solar wind but the spectral power is enhanced near the fractions of the synodic solar rotation period. This is highlighted by showing the smoothed average of the Southern and Northern Hemisphere VAI spectra (yellow lines). These spectra are consistent with the SPE analysis results showing reproduced quasiperiodic mean variations often over many years (Figs. 2-6).

The above periodicities are common in the solar wind data (e.g. Bolzan et al., 2005), they are expected in time series of geomagnetic indices (Mlynczak et al., 2008), and planetary waves have very similar periods (e.g. Jarvis, 2006; Fig. 2). Mlynczak et al. also found a very strong 9-day period in the daily global power radiated by thermospheric carbon dioxide and nitric oxide in infrared pointing to a direct solarterrestrial coupling. Periodicities of planetary waves in the upper atmosphere are similar to those in the troposphere and stratosphere (Madden, 1978) and so it is often assumed that these waves originate in the lower atmosphere and propagate upward. The results presented in this paper show the presence of solar wind periodicities in the upper-level tropospheric VAI data.

In summary, in Sect. 4 the original results by Wilcox et al. showing a link between upper-level tropospheric circulation and solar wind structure have been verified using the 
ERA-40 re-analysis vorticity (for 300- and 500-mb level). The SPE analysis of VAI time series from 1963 to August 2002 for intervals \pm 30 days from magnetic sector boundary passages is corroborated by solar wind and solar corona data. It is observed that, during late autumn and winter, the VAI is modulated by recurrent solar wind streams that have their stream interfaces near the sector boundaries and which can be traced back to the solar corona, in particular to the leading edge of coronal holes. Very similar results are found for both the Northern and Southern Hemispheres. It is shown that, apart from a mean delay of 1-2 days, the mean VAI varies in phase with waxing and waning solar wind streams indicating that there is a component of the VAI variation that is driven by the solar wind. With the exception of the Northern Hemisphere 500-mb result after 1973 during years of low aerosols, namely the missing VAI minimum near epoch day +1 , particularly when the interplanetary magnetic field turns northward, the Wilcox effect and associated mean VAI variations at other epoch days are found to persist during years of low and high volcanic aerosol loading. We believe that this is removing the requirement of aerosols as a necessary condition for a significant Wilcox effect. The IMF $B_{Z}$ appears to play a role, namely by affecting the phase of the Wilcox effect and the depth of the VAI minimum. The VAI response to a sector boundary passage associated with the north-to-south reversal of $B_{Z}$ (SBZ events) is leading by up to 2 days the VAI response to events associated with the south-to-north reversal of $B_{Z}$ (NBZ events). The latter, less geoeffective events, lead to a deeper VAI minimum and a delayed, slower growing VAI enhancement. Since the VAI is an index of extratropical storminess, we now examine the occurrence of significant extratropical storms in the context of solar wind conditions (Sect. 5). A mechanism involving auroral AGWs, which are most intense just after the arrival of high-speed solar wind, is suggested (Prikryl et al., 2009) to explain the link between solar wind and tropospheric weather.

\section{Solar wind disturbances and extratropical storms}

As discussed in the companion paper (Prikryl et al., 2009), it is known that precipitation and latent heat release are associated with mesoscale slantwise convection which is due to release of the conditional symmetric instability (Schultz and Schumacher, 1999). This instability concept is essential to the rapid development and intensification of extratropical cyclones (e.g. Kuo and Low-Nam, 1990; Kristjánsson and Thorsteinsson, 1995; Balasubramanian and Yau, 1994a, b, 1995). Thus, if auroral AGWs can initiate the convection by releasing (triggering) the symmetric instability (Prikryl et al., 2009), they may influence the growth of extratropical cyclones. For the solar wind event shown in Fig. 1, a secondary cyclogenesis (Prikryl et al., 2009) was initiated in the tail of a mid-latitude cyclone following the arrival of a steep CIR interface due to a moderately enhanced solar wind plasma
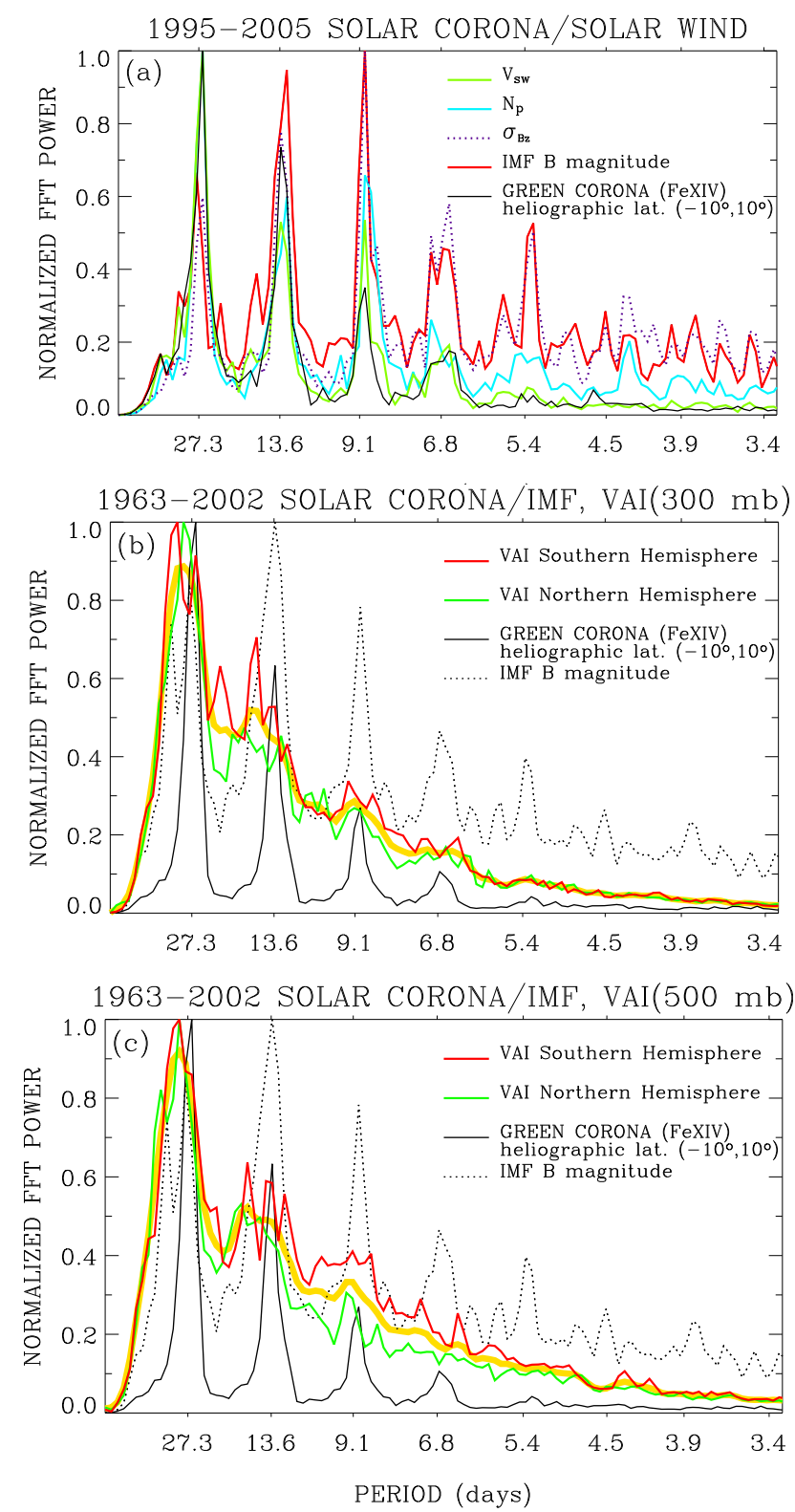

Fig. 7. Normalized averaged FFT spectra of year-long time series of solar corona intensity and (a) solar wind parameters compared with spectra of VAI at (b) 300 and (c) $500 \mathrm{mb}$. The smoothed average of the Southern and Northern Hemisphere VAI spectra are shown in yellow lines.

stream. The approximate time interval of the cyclone growth is indicated in Fig. 1a by the yellow shaded area. Another rapid cyclogenesis was initiated $\sim 24 \mathrm{~h}$ later.

The circumstantial evidence discussed in the companion paper suggests a simple approach to show a link between solar wind and the extratropical storm intensifications. First, although limited in extent, several lists of significant weather events collected in Sect. 5.1 are used to define key dates for the SPE analysis of solar wind and corona time series 


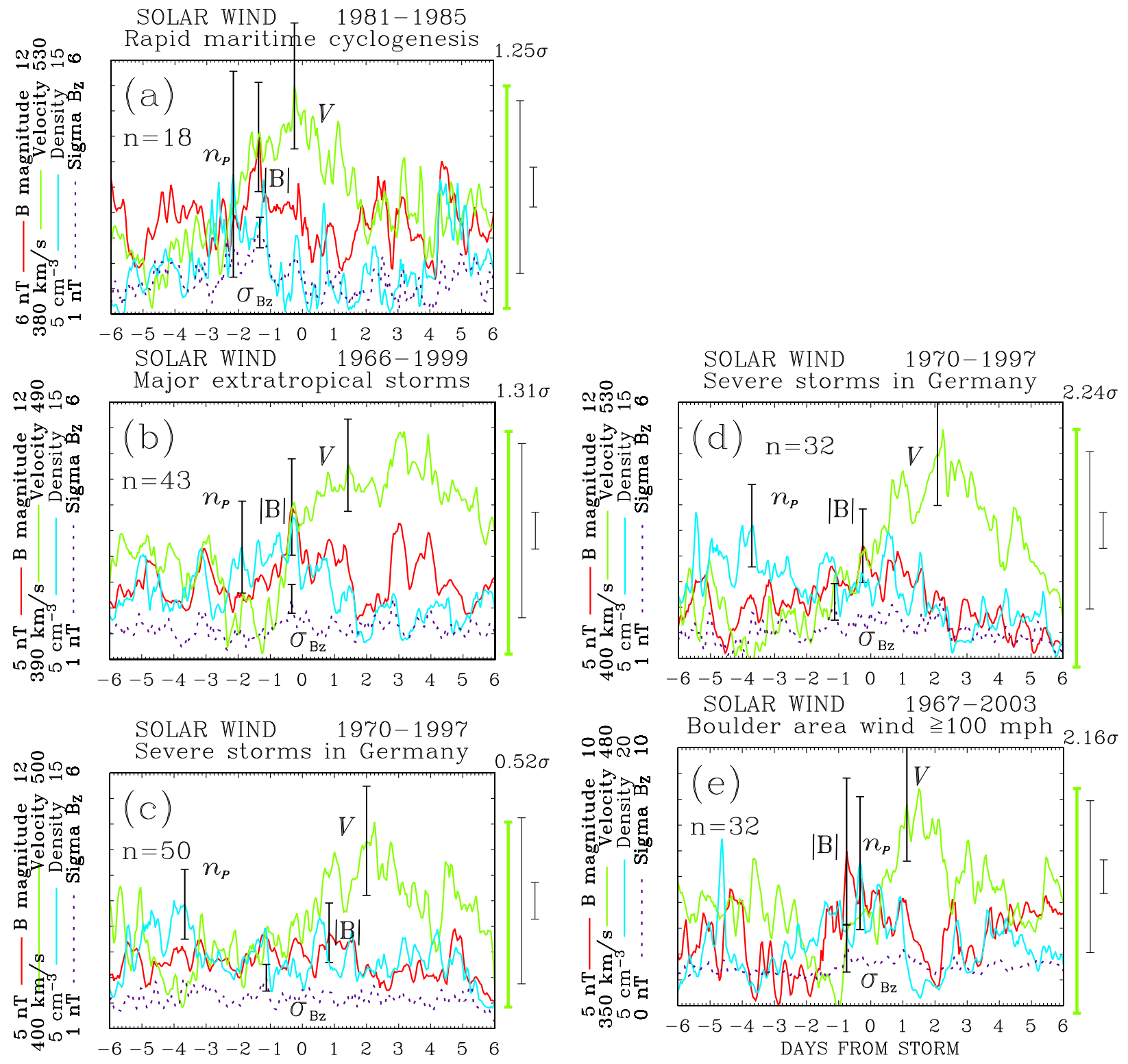

Fig. 8. The SPE analysis of solar wind velocity, density, magnetic field magnitude and standard deviation of the IMF $B_{Z}$ keyed by the start time/date of (a) explosive cyclogenesis (Davis and Emanuel, 1988), (b) major storms (Table 2), (c) 50 most intensive storm events in Germany (Klawa and Ulbrich, 2003), (d) the latter events except for those that occurred in solar wind data gaps and the events that were associated with CIRs/CMEs that occurred during the declining phase of streams, and (e) significant wind gusts greater or equal $100 \mathrm{mph}$ recorded in the Boulder region. Representative error bars plotted for each solar wind plasma parameter are twice the standard error of the mean $\sigma_{M}$. The green bar on the right is the observed mean solar wind velocity amplitude (difference between the maximum and minimum) over the 12-day period. The two bars next to it are the mean amplitude and one standard deviation $\sigma$ from the mean for 100 sets of random key times representing the statistical noise. Departures $\Delta$ of the observed mean amplitude of $V$ from the estimated statistical noise level in terms of $\sigma$ are printed in the upper-right corner of each plot (see Sect. 5.5 for details).

(Sects. 5.2-5.5). This is followed by a statistical analysis (Sect. 5.6) of a large database of storms selected by using more objective criteria.

\subsection{Severe extratropical "winter" storms in North America and Europe}

It is noted that 18 cases of moderate to strong rapid maritime cyclogenesis in the period 1981-1985, studied by Davis and Emanuel (1988, their Table 1), occurred up to 3 days after 
Table 2. Major extratropical storm events in North America and Europe.

\begin{tabular}{|c|c|c|}
\hline Date/Location & Storm name & days after(+) CIR/CME \\
\hline \multicolumn{3}{|l|}{ North America } \\
\hline 4 Mar 1966 & ${ }^{1}$ Winnipeg snowstorm of the Century & +1 \\
\hline 17-20 Apr 1967 & ${ }^{1}$ Blizzards of Southern Alberta & +1 \\
\hline 27-29 Apr 1967 & & $+4^{\mathrm{b}}$ \\
\hline 4 Mar 1971 & ${ }^{1}$ Montreal snowstorm of the Century & +1 \\
\hline 3 Apr 1974 & $1,2,3$ Super Tornado Outbreak & $+0^{\mathrm{c}}$ \\
\hline 1-2 Dec 1974 & ${ }^{4}$ Thanksgiving weekend snowstorm & $+\mathbf{0}$ \\
\hline 10 Nov 1975 & 1,2 Great Lakes storm (Edmund Fitzgerald) & $+\mathbf{0}$ \\
\hline 2 Feb 1976 & ${ }^{1}$ Groundhog Day storm & +2 \\
\hline 26-27 Jan 1978 & ${ }^{5}$ The Great Blizzard of 1978 & +1 \\
\hline 10-11 Sep 1978 & ${ }^{6}$ Queen Elizabeth II storm & $+2^{\mathrm{c}}$ \\
\hline 18-19 Feb 1979 & ${ }^{7}$ Presidents' Day cyclone & $+\mathbf{0}$ \\
\hline 13-15 Nov 1981 & ${ }^{8}$ Double windstorm & $+2^{\mathrm{c}}$ \\
\hline 15 Feb 1982 & ${ }^{1}$ Ocean Ranger Disaster & $+4^{\mathrm{c} /+1}$ \\
\hline 22-26 Feb 1982 & ${ }^{1}$ PEI Blizzard & $+\mathbf{5} /+1^{\mathrm{bc}}$ \\
\hline 29-31 Oct 1991 & ${ }^{9}$ The Perfect Storm of 1991 & $-2^{\mathrm{c}} /+\mathbf{1}^{\mathrm{c}}$ \\
\hline 10-12 Dec 1992 & 2,10 Nor' easter of 1992 & $+\mathbf{3}^{\mathrm{b}}$ \\
\hline 12-14 Mar 1993 & ${ }^{2,11}$ Superstorm'93 & $+\mathbf{3} /+1^{\mathrm{a}}$ \\
\hline 6-8 Jan 1996 & 2,12 Blizzard '96 & $+4^{\mathrm{b}}$ \\
\hline 17 Oct 1996 & ${ }^{1}$ Vancouver Island weather "bomb" & $+1 /+\mathbf{0}$ \\
\hline 24-26 Oct 1997 & ${ }^{13}$ Blizzard '97 (Nebraska storm) & $+1 /+0$ \\
\hline 7-9 Jan 1998 & ${ }^{1,2}$ Cyclone of Ice Storm of the Century & $-1 /+1^{\mathrm{c}}$ \\
\hline 15-16 May 1998 & ${ }^{2}$ Minnesota storm & $+\mathbf{0}$ \\
\hline 2-15 Jan 1999 & ${ }^{1}$ Toronto snowstorm of the Century & $-11 /-4 /-2^{c} /+5^{b}$ \\
\hline 11 Feb 1999 & ${ }^{1}$ Canadian Greatest Single-Day Snowfall & $+\mathbf{0}$ \\
\hline \multicolumn{3}{|l|}{ Europe } \\
\hline 13 Nov 1972 & ${ }^{14}$ Niedersachsen Orkan & $-2 /+1$ \\
\hline 3 Jan 1976 & ${ }^{14}$ Capella & $+\mathbf{0}^{\mathrm{a}}$ \\
\hline 24-25 Nov 1981 & ${ }^{15}$ Gale in Denmark & $-\mathbf{1}^{\mathrm{c}} /+1^{\mathrm{b}}$ \\
\hline 6-9 Nov 1982 & ${ }^{15}$ Gale in France & $?^{\mathrm{a}}$ \\
\hline 15-16 Oct 1987 & ${ }^{15,16}$ The Great Storm & $+2^{\mathrm{a}}$ \\
\hline 25 Jan 1990 & ${ }^{14,15}$ Daria/ $/{ }^{17}$ Burn's Day Storm & $+2 /+0^{\mathrm{c}}$ \\
\hline 3 Feb 1990 & 14,15 Hertha & $-1 /+2^{\mathrm{a}}$ \\
\hline 26-28 Feb 1990 & 14,15 Vivian and Wiebke & $?^{\mathrm{a}}$ \\
\hline 5-6 Jan 1991 & ${ }^{15}$ Undine & +1 \\
\hline 2-3 Feb 1991 & ${ }^{18}$ Icelandic "Greenhouse Low" & +2 \\
\hline 13 Jan 1993 & ${ }^{14}$ Verena & $+\mathbf{0}$ \\
\hline 27 Jan 1994 & ${ }^{14,15}$ Lore & +1 \\
\hline 23-25 Dec 1997 & ${ }^{15}$ Yuma & +0 \\
\hline 1-5 Jan 1998 & 15،Winter storms" & $+3 /+2^{c}$ \\
\hline 24 Oct 1998 & ${ }^{15}$ Winnie & $+\mathbf{1}^{\mathrm{bc}}$ \\
\hline 27-29 Oct 1998 & ${ }^{15}$ Xylia & $+\mathbf{0}$ \\
\hline 26-27 Dec 1998 & ${ }^{15}$ Silke & +1 \\
\hline 3-4 Dec 1999 & 14,15 Anatol & $+\mathbf{0}$ \\
\hline 25-28 Dec 1999 & $14,15,19$ Lothar and Martin & $-2^{\mathrm{c}} /+1$ \\
\hline
\end{tabular}

a Solar wind data gap; geomagnetic activity indices were used as proxies, where possible, to estimate CIR/CME arrivals, ${ }^{\mathrm{b}}$ storms that started during the declining phase of a stream and c CME events. Storm "delay" after CIR or CME is highlighted if the maximum solar wind speed exceeded $500 \mathrm{~km} / \mathrm{s}$.

(1) http://www.msc-smc.ec.gc.ca/media/top10/; (2) http://www.ncdc.noaa.gov/oa/reports/weather-events.html; (3) Agee et al. (1975); (4) http://www.crh.noaa.gov/dtx/1886-1974. php; (5) http://www.crh.noaa.gov/dtx/?page=stories/blizzard1978; (6) Gyakum (1983); (7) Bosart (1981); (8) Reed and Albright (1986); (9) http://www.ncdc.noaa.gov/oa/satellite/ satelliteseye/cyclones/cyclones.html; (10) Bresky and Colucci (1996); (11) Dickinson et al. (1997); (12) http://www.cpc.ncep.noaa.gov/products/special_summaries/96_1/; (13) http://www.crh.noaa.gov/pub/blizzard97/blizzard97.php; (14) Klawa and Ulbrich (2003); (15) http://www.abi.org.uk/Display/File/78/windstorm_report.pdf; (16) Burt and Mansfield (1988); (17) MacCallum (1990); (18) Kristjánsson and Thorsteinsson (1995); (19) Wernli et al. (2002) 
CIRs in the solar wind. The absence of solar wind plasma data in 4 cases prevented firm identification of CIRs, but for at least three of these cases the IMF magnitude and the AE index were enhanced and went through a peak. These observations were consistent with the CIR arrival just before the tropospheric event. As mentioned above, CIRs dominate the solar wind interaction with the magnetosphere during the declining phase of the solar cycle, such as the period 19811985.

We find a strong tendency for major storms to occur within 2 days of the arrival of a high speed solar wind. Table 2 lists major extratropical storms that include the most severe autumn-winter windstorms and snowstorms based on the MSC and NOAA resources on the internet and official published reports (e.g. ABI, 2003; Klawa and Ulbrich, 2003) of major storm damage, economic loss and loss of life (Kunkel et al., 1999). Some references to papers on detailed analysis of specific major storms are also included in Table 2.

The major storms that are listed in Table 2 are relatively rare events that occurred over a period of more than 30 years. A comprehensive and homogeneous database of such events over such long period, if it exists, has not been available to us. Thus we have had to rely on published literature and reports. To minimize the subjectivity of our selection, we have tried to find lists or subsets of major storms objectively selected and to use them as given. One example is a list of moderate to strong rapid cyclogenesis events compiled by Davis and Emanuel (1988). All European storms in Table 2 except for the Icelandic "Greenhouse Low" are from a report by the ABI insurance company and one other publication quoting losses according to another insurance company, Munich Re (Klawa and Ulbrich, 2003, see Table 2 therein). Summer storms are excluded from this list. The selection of these storms was based upon the extensive damages and insured losses that they caused (ABI, Munich Re). We have not found a similar list of major extratropical storms for North America and thus we have had to use resources scattered in the literature or the Internet. However, 13 events (major winter storms before 2000) were taken from one source (1) although other references are added for more detail. While the rest of the storms are gathered from various sources, they are all major events similar to those that occurred in Europe, and with about the same occurrence rate.

To complement the analysis, a list of 50 severe winter storms in Germany objectively selected by Klawa and U1brich (2003, Table 4) is used in Fig. 8c and a subset of these storms in Fig. 8d. A similar set of objectively selected significant local events are wind gust measurements in the Boulder region, Colorado (http://www.cdc.noaa.gov/Boulder/). In this case, a simple criterion is used, namely the selection of days when the observed wind gusts exceeded a given threshold ( $\geq 100 \mathrm{mph}$ ).

The third column in Table 2 gives days of CIRs or CMEs relative to the start date of the storm. In most cases, only the day of the closest solar wind event that preceded the storm is given. In some cases, two or more solar wind events are given, particularly when storms lasted for several days. Most of the storms occurred (started) within 2 days of CIR or CME arrivals (see, footnotes $\mathrm{a}, \mathrm{b}$, and $\mathrm{c}$ in Table 2). The storm delays that are highlighted are for interaction regions (either CIR or CME) that preceded the fast solar wind that exceeded $500 \mathrm{~km} / \mathrm{s}$.

Superstorm'93 started 3 days after a strong CIR was observed just before a gap in solar wind data. It was associated with high AE-index peak hourly values of up to $\sim 800 \mathrm{nT}$ ( $K_{p}$ index 7-) on 8/9 March. The AE index dropped below $300 \mathrm{nT}$ the next day, but peaked at $1246 \mathrm{nT}$ on 11 March possibly due to another CIR one day before Superstorm'93. The high geomagnetic activity continued for several days, suggesting a structured, very intense and long-lasting (wide) stream. The UK Great Storm 1987 occurred just after a solar wind data gap when the solar wind speed $V$ had already reached nearly $800 \mathrm{~km} / \mathrm{s}$ and a geomagnetic storm was in progress; the AE-index peaked at $954 \mathrm{nT}$ two days earlier, most likely due to a CIR arrival. These and some other storms occurred during solar wind data gaps (footnote a).

Some of the storms started during the declining phase of a stream (footnote b) when the solar wind fluctuations were Alfvénic and lasted for several days. For example, the first of a series of January 1999 storms occurred after the solar wind Alfvén waves coupled to the magnetosphereionosphere and generated intense ionospheric convection pulses and large amplitude TIDs (similar to cases discussed by Prikryl et al., 2009) that were observed for several hours starting $\sim 16: 00$ UT on 1 January. The archive of GOES images indicates that a cyclogenesis occurred southwest of the Great Lakes region on 2 January. This cyclone resulted in the first snowstorm that affected the region in January. It was followed by a series of significant cyclones that affected the Great Lakes region on 6-7, 8-9, 13, and 14-15 January. The development of these cyclones was associated with arrivals of solar wind streams on 6 and 13 January, i.e. 4 and 11 days after the start day of the "Toronto snowstorm of the Century" (Table 2).

\subsection{The SPE analysis of solar wind}

The SPE analysis is applied to the solar wind data to verify the suggested association between high-speed solar wind and the occurrence of these severe storms. Rather than using solar wind events as key times as in Figs. 2-6, the solar wind time series are now keyed by the start time/date of the storm. Figure 8 shows the averaged solar wind data for: (a) 18 moderate to strong explosive cyclogenesis events (Davis and Emanuel, 1988, their Table 1), (b) 43 major winter storms in North America and Europe (Table 2), (c) 50 most intensive storm events in Germany (Klawa and Ulbrich, 2003), (d) a subset of the latter events, and (e) significant wind gusts recorded in the Boulder region. The gaps in solar wind data such as those mentioned above reduce the actual 
number $\mathrm{n}$ for a given epoch day (hour). The SPE analysis results for these storm groups and their statistical significance are now briefly discussed.

For the first storm group (Fig. 8a), the mean CIR near day -2 at the leading edge of the mean stream indicates that, on average, these events were initiated $\sim 2$ days after the streams arrivals. The mean standard deviation $\sigma_{B z}$ also peaks just prior to day -1 . For the group of major winter storms (Fig. 8b) the solar wind velocity $V$ goes through a minimum near day -1 while the proton density $n_{p}$ and the IMF magnitude $|B|$ both peak just before day 0 . Therefore, on average, these storms occurred $\sim 1$ day after a stream or a CME arrival. Representative standard errors of the mean $\left( \pm 1 \sigma_{M}\right)$ near the maximum for each parameter are shown. The excursion of the mean solar wind velocity (difference between the maximum and minimum $V$ ) exceeds $4 \sigma_{M}$. An excursion (amplitude) that is greater than $2 \sigma_{M}$ indicates a statistical significance at $95 \%$ or above. While the numbers of storms in individual storm groups are relatively small and thus the $\sigma_{M}$ values are large, the mean solar wind parameters show temporal patterns that are very similar to those shown for SBP events (e.g. Fig. 2b). Also, the separate SPE analysis results for North American and European storms (not shown) are similar and show statistically significant and reproducible patterns, particularly for the solar wind velocity $V$. Another method using randomly selected key dates to estimate the statistical significance of the SPE analysis is discussed in Sect. 5.5.

To further verify the reproducibility of the storm results shown in Fig. 8a and b the SPE analysis is applied to other independent lists of storms. Klawa and Ulbrich (2003, see their Table 4) identified severe winter storm events in Germany to model the estimation of storm losses. They ranked 50 of the most intensive storm events for the period 19701997 according to their loss model. Most of the events that are ranked in the top 10 have already been included in Table 2 although some of these events occurred during solar wind data gaps.

Figure 8c shows the SPE analysis of solar wind data for all 50 events of Klawa and Ulbrich (2003). Although the peak distributions in $|B|$ and $n_{p}$ are spread over several days and do not show a distinct average peaks prior to day 0 , the mean solar wind velocity increases to a maximum after the key day. The amplitude (excursion) of $V$ is greater than two standard errors of the mean and thus statistically significant at or above the $95 \%$ confidence level. It should be noted that 9 (out of 50) storms occurred during solar wind data gaps and thus any sparse solar wind data during these intervals do not significantly contribute to the SPE analysis result shown in Fig. 8c. Another 9 storms were associated with CIRs (within 2 days of their arrivals) which occurred during the declining phase of structured streams. The SPE analysis of solar wind data for these 9 storms separately results in a generally downward trend in solar wind velocity after a broad maximum around -3 days (not shown), thus reducing the mean amplitude of the velocity pattern in Fig. 8c. For the rest of the events, 3 events occurred 3 days after and 29 events up to 2 days after a CIR on the leading edge of a stream. In Fig. 8d, the SPE analysis for these 32 events produces a significantly enhanced velocity pattern which is comparable to the major storm result (Fig. 8b).

Finally, a list of significant wind events that occurred in the Boulder area of Colorado from 1967 to 2003 is used (http://www.cdc.noaa.gov/Boulder/). As mentioned on the website, Boulder has some of the highest peak winds of any city in the United States. The wind gust database gives the date and, in some cases, the approximate time of peak gusts in mph units; gusts greater or equal to $90 \mathrm{mph}$ are highlighted. The results of the SPE analysis of solar wind parameters using the Boulder wind events with gusts greater or equal $100 \mathrm{mph}(45 \mathrm{~m} / \mathrm{s})$ are shown in Fig. 8e. The mean solar wind velocity has a well defined minimum one day before the key day and reaches a maximum 2-3 days later $(+1.5$ epoch days). The other solar wind plasma parameters peak just before key day 0 but are enhanced over \pm 1 epoch days. This is the average duration of the interaction region at the leading edge of fast solar wind. The amplitude of the mean excursion (difference between the maximum and minimum) for all plasma parameters is between $\sim 1 \sigma_{M}$ and $4 \sigma_{M}$. Very similar results are obtained for peak gust threshold of $90 \mathrm{mph}$ (59 events) although the amplitudes of the mean parameter variations and the standard errors of the mean are approximately one half of those in Fig. 8e.

Figure 9 shows the temporal occurrence distribution of CIR/CMEs with respect to the start time of the storm for the same storm groups as shown in Fig. 8. CIRs and CME shocks that preceded the fast solar wind in excess of $500 \mathrm{~km} / \mathrm{s}$ are shown in black. These distributions show that the majority of CIRs/CMEs occurred a few days before or on the day of the storm.

\subsection{The SPE analysis of solar corona}

The SPE analysis of the green coronal intensity is performed for storms used in Fig. 8a-e, except for those that were closely preceded by CMEs. For each storm group, the averaged synoptic maps (Fig. 10a-e) show a coronal hole prior to the key day and preceding the mean solar wind stream. All these storms, with duplicates among the groups eliminated, are then combined in the SPE analysis of green corona and solar wind in Fig. 11. The solar corona result (Fig. 11a) shows a smoother mean coronal hole centered 2-3 days prior to the start day of storm. The solar-wind parameters (Fig. 11b) show the corresponding mean stream preceded by superposed CIRs.

These results reinforce the observation for each storm group that there is a tendency of such storms to occur within a few days of arrival of high-speed solar wind from coronal holes. This is consistent with the correlation between 


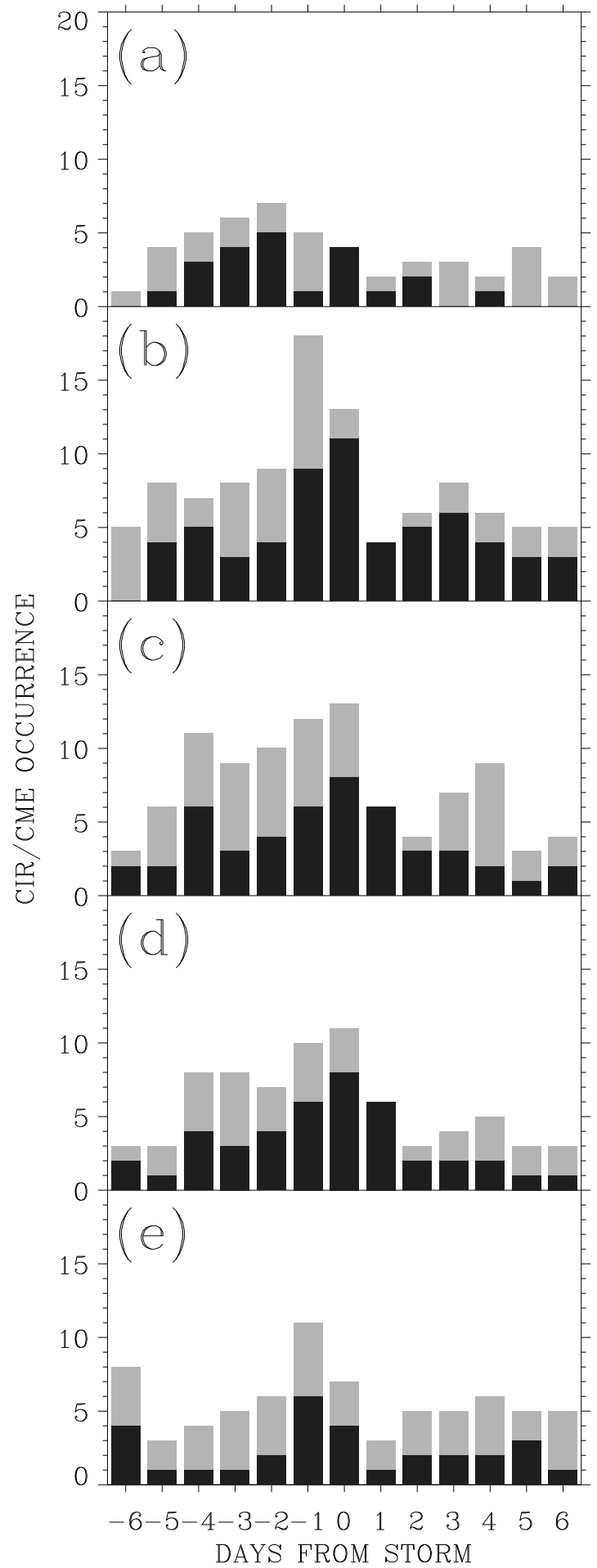

Fig. 9. The distribution of CIR/CMEs arrival times relative to the date of the storm (day 0 ). The subset distributions of arrivals of major streams with a maximum velocity in excess of $500 \mathrm{~km} / \mathrm{s}$ are shown in black. Minor CIR/CMEs are shown in grey. Panels (a-e) correspond to those in Fig. 8. the mean VAI and coronal intensity and/or solar wind sector structure (Figs. 2-6).

\subsection{Other types of storms}

Now we briefly discuss special types of extratropical cyclones in the context of the solar wind. As pointed out by Thorpe (2002), secondary cyclones spawned from the frontal zones of the primary cyclones are not well understood and are difficult to forecast. He suggested that these secondary cyclones require a conceptual model that may be very different from the primary ones. The Case- 2 extratropical cyclone (Prikryl et al., 2009) was initiated by a secondary cyclogenesis in the tail (frontal zone) of the previous, primary one, after a CIR arrival (Fig. 1a).

Heyraud et al. (2004) selected 6 cases of cyclones from the Fronts and Atlantic Storm-Track Experiment (FASTEX) for study of mesoscale processes and the energetics of secondary cyclogenesis. All 6 events occurred within 2 days of the CIR arrivals ( 4 events within 1 day of strong CIRs). Other secondary cyclogenesis events (e.g. Carrera et al., 1999; Lemaître et al., 1999) were also closely preceded by CIRs.

Most of the explosive cyclogenesis events in the westcentral North Atlantic Ocean during 1981-1984 that were studied by Sanders (1986) have been included in the study by Davis and Emanuel (1988). Those that are not included in Fig. 8a also show a tendency to occur in association with CIRs or CMEs, although there are significant solar wind data gaps in 1982-1984. Meteorological precursors to explosive and ordinary cyclogenesis were studied by Gyakum and Danielson (2000) for cases of cold season cyclogenesis from 1975 to 1995 in the western North Pacific Ocean. Using the times of "strong (moderate bomb)" and "weak (strong nonbomb)" events from their Table 1 (37 events in total), we find a strong tendency supported by the SPE analysis for both types of events to occur in association with solar wind disturbances. A similar trend toward rapid development in extratropical cyclones following the arrival of solar wind CIRs is found for "striated" cloud systems (Feren, 1995, private communication, 2007) or striated cloud heads observed in satellite images (Dixon et al., 2000).

These observations and results (Figs. 8-11) strongly suggest a link between solar wind disturbances and tropospheric weather. They are consistent with previously observed solar wind influence on extratropical storminess and vorticity (VAI) as discussed in Sect. 4. It is suggested that the enhanced auroral AGWs resulting from solar wind disturbances may interact with evolving weather systems and play a role in destabilizing the atmosphere by initiating convection, and possibly contributing to the development of the baroclinic instability. 

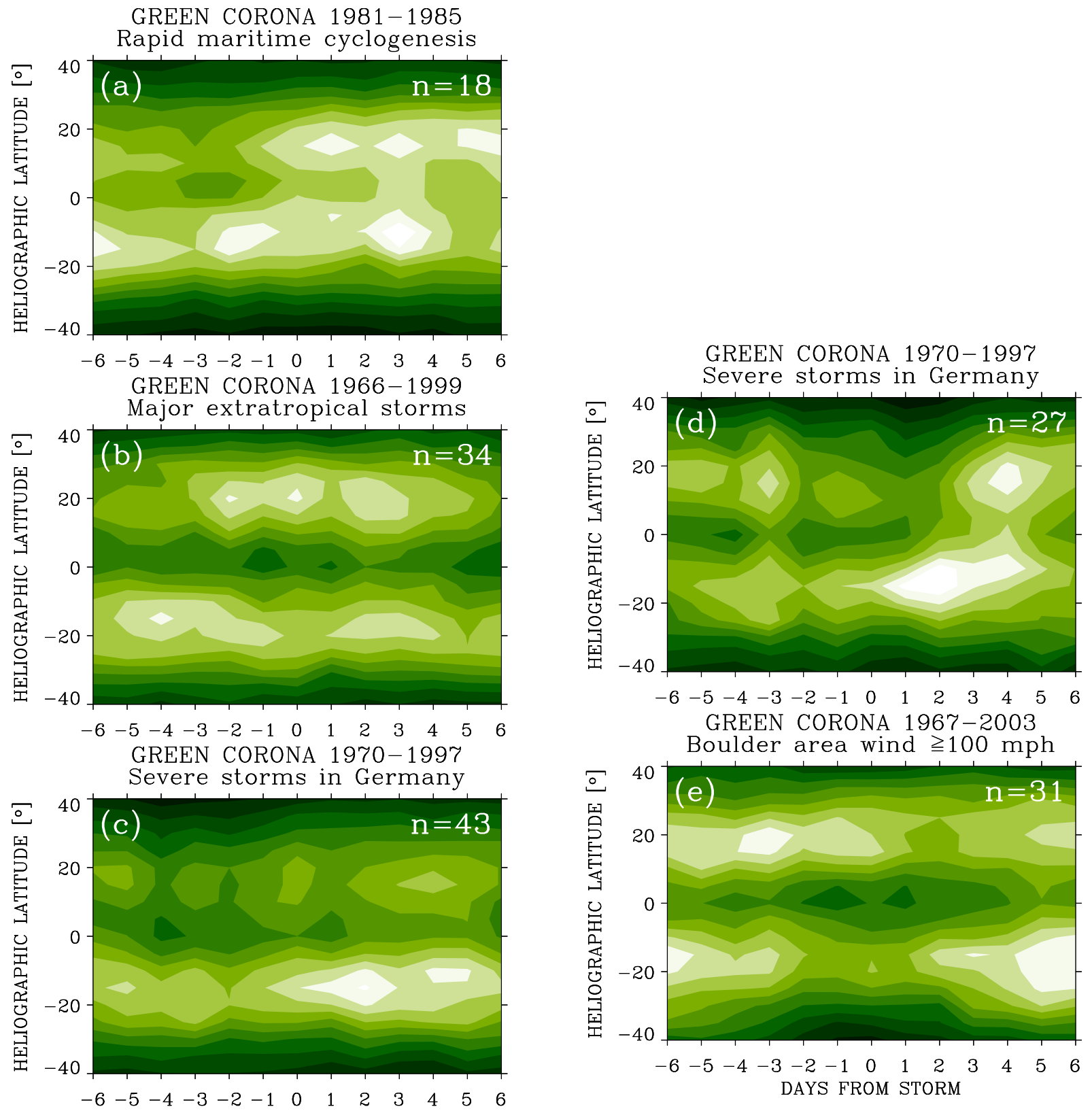

Fig. 10. (a-e) Superposed epoch analysis of the green corona keyed by the time/date of extratropical storm. Panels (a-e) correspond to those in Fig. 8. Storms that were closely preceded by a CME are excluded. The intensity contours are (a) 5 coronal units and (b), (c), (d), (e) 3 coronal units apart.

\subsection{Statistical significance of the SPE analysis results}

Although the standard errors of the mean and reproducibility of the temporal variations of the solar wind plasma parameters, $V$ in particular, indicate that the SPE analysis results discussed above have statistical significance at the $95 \%$ level or above, this could be an overestimate (Shapiro, 1976; Hines and Halevy, 1977; Williams, 1978; Haurwitz and
Brier, 1981). To further assess the statistical significance of the SPE results for storms a randomization procedure is used.

We followed the approach adopted by Hines and Halevy (1977) and Williams (1978) and applied it to the SPE analysis of solar wind velocity using the storm days as key times. The (double) amplitude or excursion of the obtained mean temporal variation is defined as the difference between the maximum and minimum $V$ over 12 days. Hereafter called 

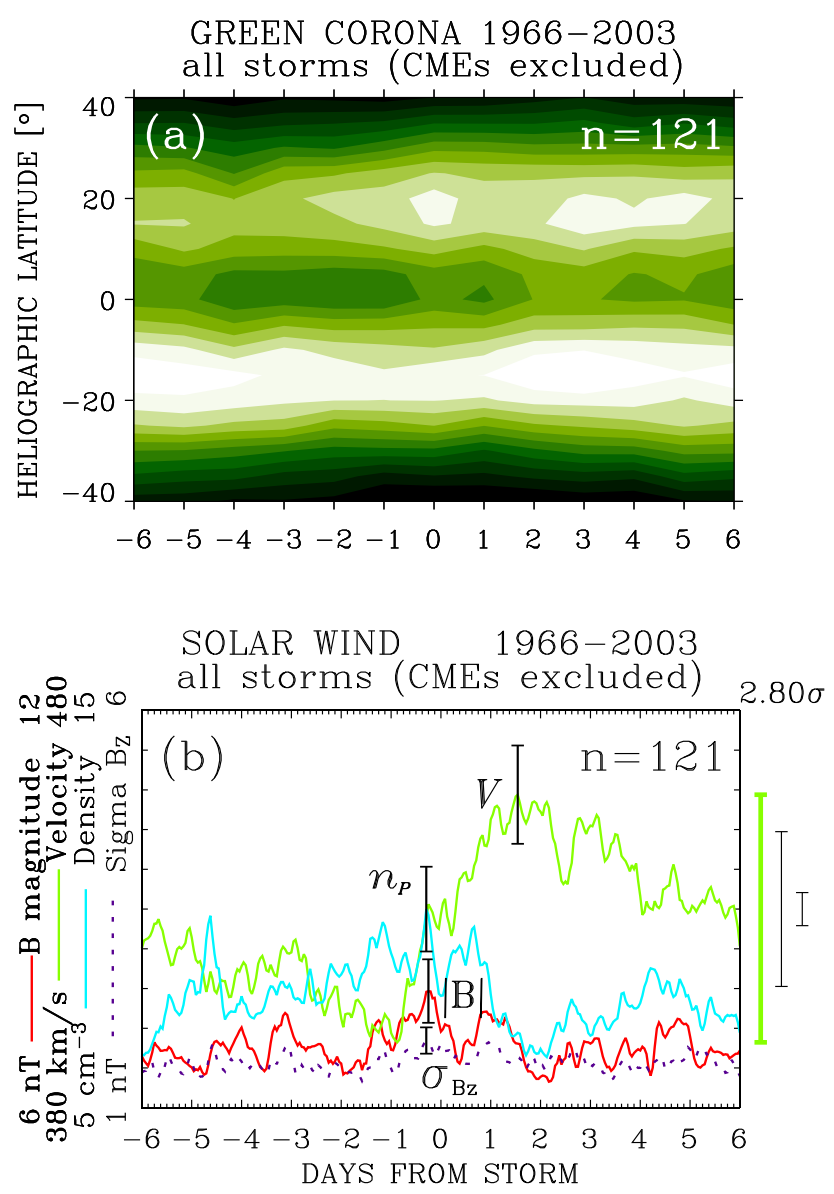

Fig. 11. The superposed epoch analysis of (a) the green solar corona emission line intensity interpolated for the central meridian and (b) solar wind plasma parameters. The time series are keyed by the time/date of extratropical storms used in Fig. 10a-e (duplicate storms are eliminated). Storms that were closely preceded by a CME are excluded. The intensity contours are 2 coronal units apart. Representative error bars plotted for each solar wind plasma parameter are twice the standard error of the mean $\sigma_{M}$. The green bar on the right is the observed mean solar wind velocity amplitude (difference between the maximum and minimum) over the 12-day period. The two bars next to it are the mean amplitude and one standard deviation $\sigma$ from the mean for 100 sets of random key times representing the statistical noise. The observed mean amplitude of $V$ exceeded the estimated statistical noise level by $2.80 \sigma$.

the mean amplitude, it is shown as a green bar on the right of each plot in Fig. 8a-e and is found to exceed two standard errors of the mean as discussed above. To further estimate the statistical significance of this amplitude, the SPE analysis is repeated for each storm group for ensembles of $n$ randomly generated key times separated by at least 4 days. This is then repeated 100 times for each storm group. The SPE analyses using 100 sets of random key times produce 100 values of amplitudes (excursions) of the mean solar wind velocity. The mean amplitude and standard deviation $\sigma$ from the mean are computed from 100 sets of random key times for each group of storms. These values that are shown as two black bars for each group of storms (Fig. 8a-e; right) represent the mean statistical noise level affecting the results of the SPE analysis of solar wind velocity using a given set of key times.

For comparison, the amplitude of the observed mean temporal variation of $V$ for key times defined by storm times is shown as a green bar. The difference $\Delta$ between the observed maximum amplitude for the solar-wind CIR key times and the mean statistical-noise-related excursion for the randomly generated key times is shown in terms of $\sigma$. If $\Delta$ exceeded the mean excursion by more than $2 \sigma$ the result is considered statistically significant at the $95 \%$ level or above (Williams, 1978). This is the case in Fig. 8d-e and Fig. 11b but not in Fig. 8a-c, although the maximum amplitude is always greater than $2 \sigma_{M}$. However, it should be noted that the maxima of the solar wind parameters determined from the SPE analyses using 100 sets of random key times are randomly distributed over the 12-day period. In contrast, when the key times are defined as storm times, the solar wind velocity maximizes just after or near the key time, i.e. 2-3 days after the mean coronal hole crossed the central meridian on the sun. The minimum of $V$ approximately falls at the epoch day of the mean coronal hole and precedes a cluster of peaks for the rest of the solar wind parameters (mean CIR). This is observed for each storm group as well as all storms combined (Fig. 11), which provides additional support for the statistical significance of the results even though other estimates may imply that the pattern amplitudes are statistically less significant than the $95 \%$ level. Thus the SPE analysis results indeed imply that severe extratropical storms tend to occur within a few days of the arrival of solar wind disturbances.

\subsection{The extratropical storm track database derived from the NCEP/NCAR Reanalysis}

Chandler and Jonas (1999) used an algorithm to identify and track sea level pressure (SLP) minima. They used raw data from the NCEP/NCAR Reanalysis from 1961 to 1998 and computed 12-hourly SLP fields, gridded to $2^{\circ} \times 2.5^{\circ}$ in latitude and longitude, respectively. We use the on-line storm track data by Chandler and Jonas to determine the start of the intensification phase of extratropical storms. First, the storms are selected by their maximum strength (the lowest SLP minimum) reached within a given geographical area. Second, the start time of the first 24-h deepening rate of the low that exceeded 0.3 Bergeron (1 Bergeron $=24 \mathrm{mb} \times \sin \phi / \sin 60^{\circ}$ ) is recorded. The deepening rate is adjusted for latitude $\phi$. It is calculated over successive (overlapping) 24-h intervals for each storm track. Because of the large grid cells of the database this is underestimating the actual deepening rate of storms but it serves as a convenient and objective criterion for selection of significant storms. The storm SLP and the deepening rate time series for a given ensemble of storms are superposed to obtain the storm ensemble averages of deepening 
curves. Thus obtained storm start times are then used as key times in the SPE analysis of solar wind data.

To support the results that were obtained so far using incomplete lists of storms we now focus on three geographical sectors in the Northern Hemisphere that include two major storm tracks spanning the eastern Pacific and west coast of North America (E190-240 E), the east coast of North America and western Atlantic $\left(280-320^{\circ} \mathrm{E}\right)$, as well as the storm track sector of the eastern Atlantic and western Europe $\left(320-10^{\circ} \mathrm{E}\right)$. A band of latitudes between $40^{\circ}$ and $60^{\circ} \mathrm{N}$ is used in all three cases to limit the locations of the maximum strength of the selected storms. The Wilcox effect on upper-level vorticity was found to have the largest amplitude at these latitudes (Wilcox et al., 1974). The above described criteria are used to select the storms from the database, determine the start times of the storm intensifications and to construct mean deepening curves for each storm ensemble. Various thresholds for SLP minima were used but here we show the results for storms that deepened to their overall minimum below $970 \mathrm{mb}$ within a given sector.

Using the start times of the storms as key times, the SPE analysis results are shown in Figs. 12-14 for (a) the solar wind parameters and (b) the mean SLP minima and the 24-h deepening rate for each storm ensemble for which the storm tracks are shown (c). Locations of the first deepening that exceeded the threshold of 0.3 Bergeron is marked by green points while the lowest overall SLP minima for each storm that were within a given sector are shown as red points. The selection criterion rendered quite consistent mean deepening curves for each storm ensemble. A 2-day wide peak in the deepening rate marks a relatively steep section of the mean deepening curve that reaches a minimum about 2 days from the start (day 0). The dotted lines show the extent of two standard errors of the mean that is very small for the deepening phase where the threshold condition of 0.3 Bergeron is applied to determine the storm start time and the number of values averaged were exactly or very close to the number of storms. The errors increase at the extremities of the curves as the number of values averaged drops due to smaller number of long storm tracks.

The results for the SPE analysis of solar wind plasma parameters are very similar to those already shown in Figs. 8 and 11. The standard errors of the mean $\left( \pm 1 \sigma_{M}\right)$ that are shown for the solar wind data are representative for the whole epoch interval. The number of hourly values averaged for each epoch hour varies since the data gaps reduce the number of values entering the averages to about $60-70 \%$ of the actual number of storms. The solar wind data are available from 27 November 1963 thus only storms that occurred after this date are used. Consistent with Figs. 8 and 11, for each storm ensemble the mean solar wind velocity increases 1-2 days prior to the storm start and remains elevated during the deepening phase of the storm. The other plasma parameters are elevated and peak prior to the storm start. As the mean solar wind velocity starts to increase about one day before

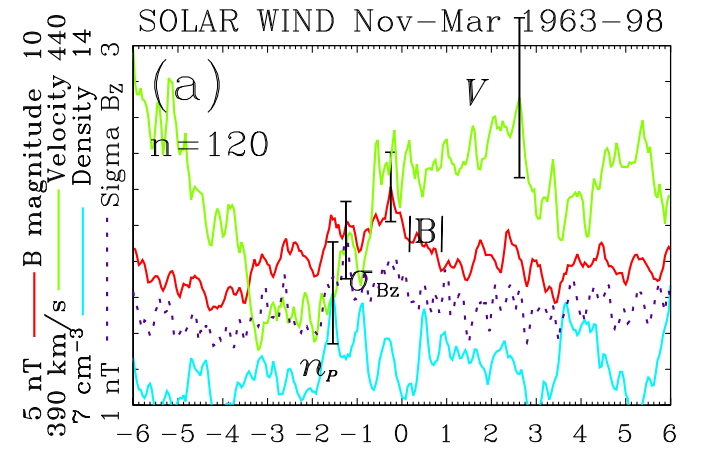

STORMS $<970 \mathrm{mb}>0.3$ Bergeron

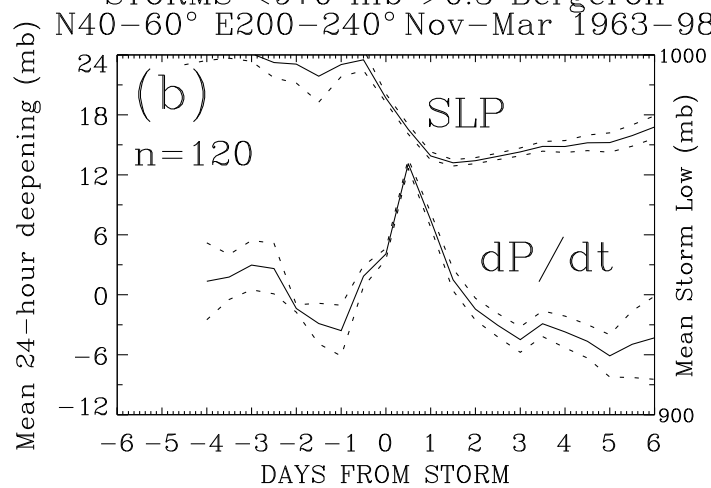

DAYS FROM STORM

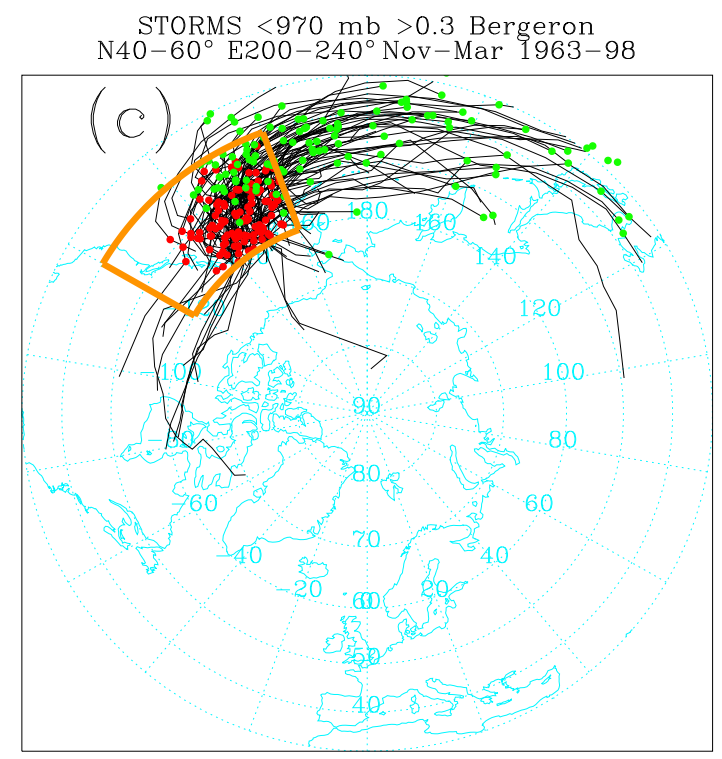

Fig. 12. The superposed epoch analysis of (a) solar wind plasma parameters and (b) the storm SLP minima and the deepening rate for the north-eastern Pacific storms. The time series are keyed by the start time of storms. (c) Storm tracks (black), locations of the storm first deepening greater than 0.3 Bergeron (green), and the storm lowest SLP (red) that was reached within the geographic sector (orange), are shown. 

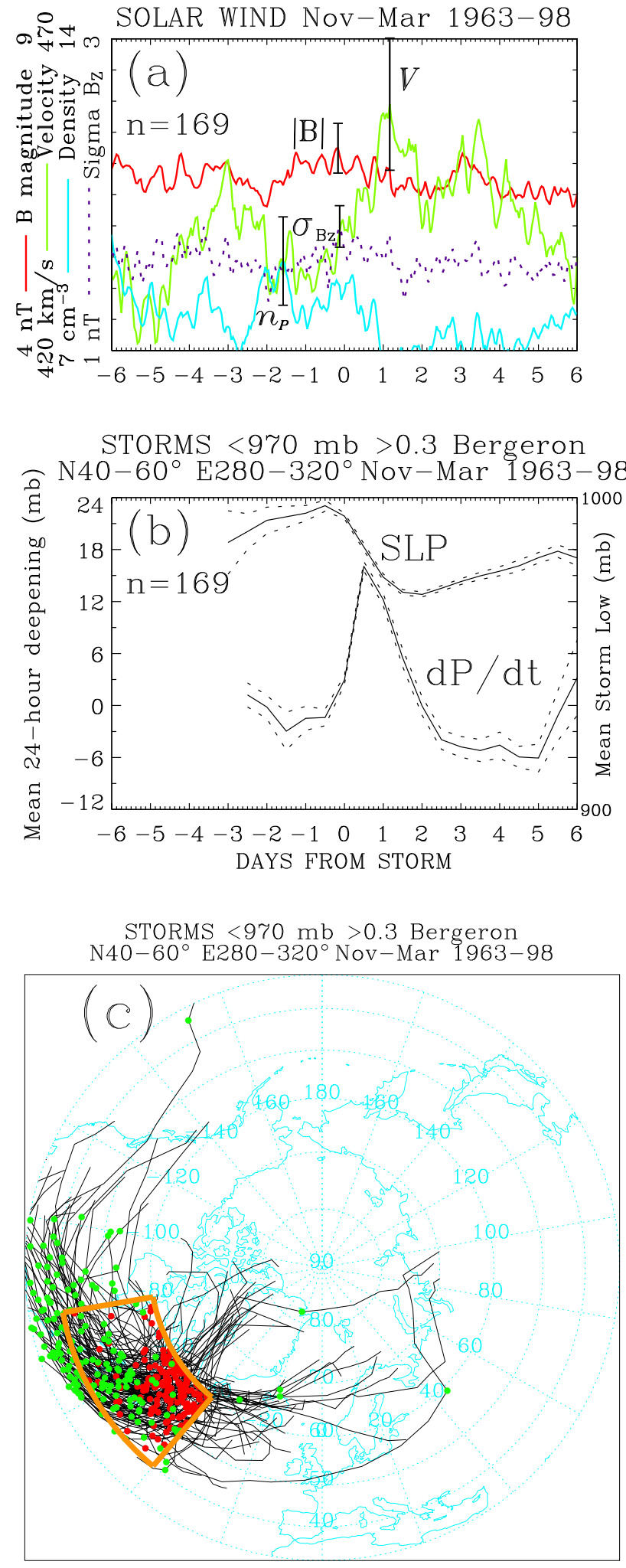

Fig. 13. The same as Fig. 12 but for the storm sector spanning the east coast of North America and west Atlantic. the storm start there are enhancements in the proton density, magnetic field magnitude as well as the amplitude of the IMF $B_{Z}$ component fluctuations $\sigma_{B z}$. While broadened due to varying time offset of the storm with respect to the arrival of the fast solar wind, this pattern is a result of a superposition of stream interaction regions at the interface between the fast and slow solar wind from coronal holes or CMEs. The storm intervals when CMEs occurred near the storm start were not removed from the analysis as done in Fig. 11. The error bars are reduced and the trends smoothed out by including all three storm sectors in the combined SPE analysis that significantly increases the number of superposed epochs (Fig. 15). Figure $15 \mathrm{~b}$ shows the IMF $B_{Z}$ component, the standard deviation $\sigma_{B z}$ and the quasi-invariant index QI that combines three plasma parameters (see Sect. 3). In Fig. $15 \mathrm{a}, \sigma_{B z}$ is replaced by the standard deviation of the field vector $\sigma_{B}$, which is greater than $\sigma_{B z}$ and the mean value shows a larger amplitude increase near day -2 . Note that a 3-point smoothing was applied only in the top panel (a) to avoid crowding. The mean IMF $B_{Z}$ starts to fluctuate at about the same time when all the plasma parameters start to increase. This is contrasted by small amplitude fluctuations about zero and no mean trend of the IMF $B_{Z}$ before day -2 . The mean QI index peaks just prior day -1 , after going through a broad minimum a few days earlier. The pattern of the mean solar wind velocity decrease followed by an increase that is preceded by peaks in $B, n_{p}, \sigma_{B}$ and QI has the amplitude reduced by about half when no threshold for minimum SLP is applied. The amplitude increases for the strongest storms (thresholds of $960 \mathrm{mb}$ or less) when the results become comparable to those shown for severe storms in Figs. 8 and 10. The pattern persists if the storm data are split into years of high and low volcanic aerosols. The pattern disappears if the key times are randomized or if wrong years are used with storm dates.

\section{Summary of the analyses}

A three-pronged search for evidence of the solar wind influence on extratropical cyclones has been pursued.

First, the 12-hourly time series of upper-level vorticity data extracted for the period of 1963-1973 from the ECMWF ERA-40 re-analysis dataset in conjunction with the OMNI solar wind database are used to verify the original Wilcox finding. For the Northern and Southern Hemispheres, the Wilcox effect is found to be independent of the stratospheric aerosol loading that was previously suggested as a necessary condition for the effect to be observed. For the extended period of 1963-2002, the effect continues to persist through years of high and low volcanic aerosol loading except for the Northern Hemisphere at the 500-mb level after 1973 during years of low aerosol loading, when the VAI minimum just after the sector boundary passage is not found. This is consistent with previous results by Tinsley et al. (1994) and Kirkland et al. (1996). We find that this negative result in the 
absence of aerosol mainly applies to those SBP events that are associated with northward shift of the IMF $B_{Z}$. Even in that case, at other epoch times the mean VAI variations are reproduced during low and high aerosol loading and are in phase with those at $300 \mathrm{mb}$. The solar wind results are corroborated by the analysis of green corona data, which clearly show the mean recurrent coronal holes that are associated with mean VAI variations. That association suggests a modulation of VAI by solar wind streams. In this modulation the IMF $B_{Z}$ plays a role by affecting the phase of the Wilcox effect and the depth of the VAI minimum. The mean VAI response to north-to-south reversals of $B_{Z}$ is leading by up to 2 days the mean VAI response to the south-to-north reversals of $B_{Z}$. The phase shift between the mean VAI responses obtained for these two cases may explain the reduced amplitude of the overall Wilcox effect.

The spectral analysis of time series reveals periodicities in VAI data that are similar to those found in solar wind (and corona), which are the solar synodic rotation period and its fractions. While periods of planetary waves can differ from these (e.g., 16 days, which also appears in VAI data), they can be very close to some of the solar rotation fractions (e.g. 5, 9 and 13 days).

Second, it is observed that rapid cyclogenesis and severe extratropical storms including windstorms tend to occur within a few days of the arrival of CIRs at the leading edge of streams from coronal holes. These initial findings based on lists of significant storm events are supported by a statistical analysis of an extensive database of storms. It is found that, on average, the solar wind velocity starts to increase 1-2 days prior to the storm start while the other solar wind plasma parameters reach a peak. On average, the solar wind velocity reaches a maximum about 2 days after the CIR, and the auroral activity remains enhanced for a few days afterwards.

Third, in the companion paper (Prikryl et al., 2009), the link between the solar wind and extratropical cyclones is proposed to be auroral AGWs generated through coupling of solar wind MHD waves to the magnetosphere-ionosphere system. Quasi-periodic mesoscale cloud/rain bands are observed a few hours after auroral AGWs are launched and this is supported by AGW ray tracing results. It is proposed that a gravity-wave-induced vertical lift modulates the slantwise convection by releasing the moist symmetric instability in the warm frontal zone of the cyclone. Although the wave amplitude is likely small, the auroral AGW-induced lift may be just enough to release the symmetric instability and thus initiate the convection, particularly if combined with an upward tilting flow near the warm front that is near the threshold for release of the symmetric instability. It is suggested that the cumulative increase of latent heat release due to the slantwise convection initiated by auroral AGWs may lead to storminess.

A statistical increase of high-level cloudiness (International Satellite Cloud Climatology Project ISCCP-D1 dataset) in the mid-latitude storm sectors a few days after
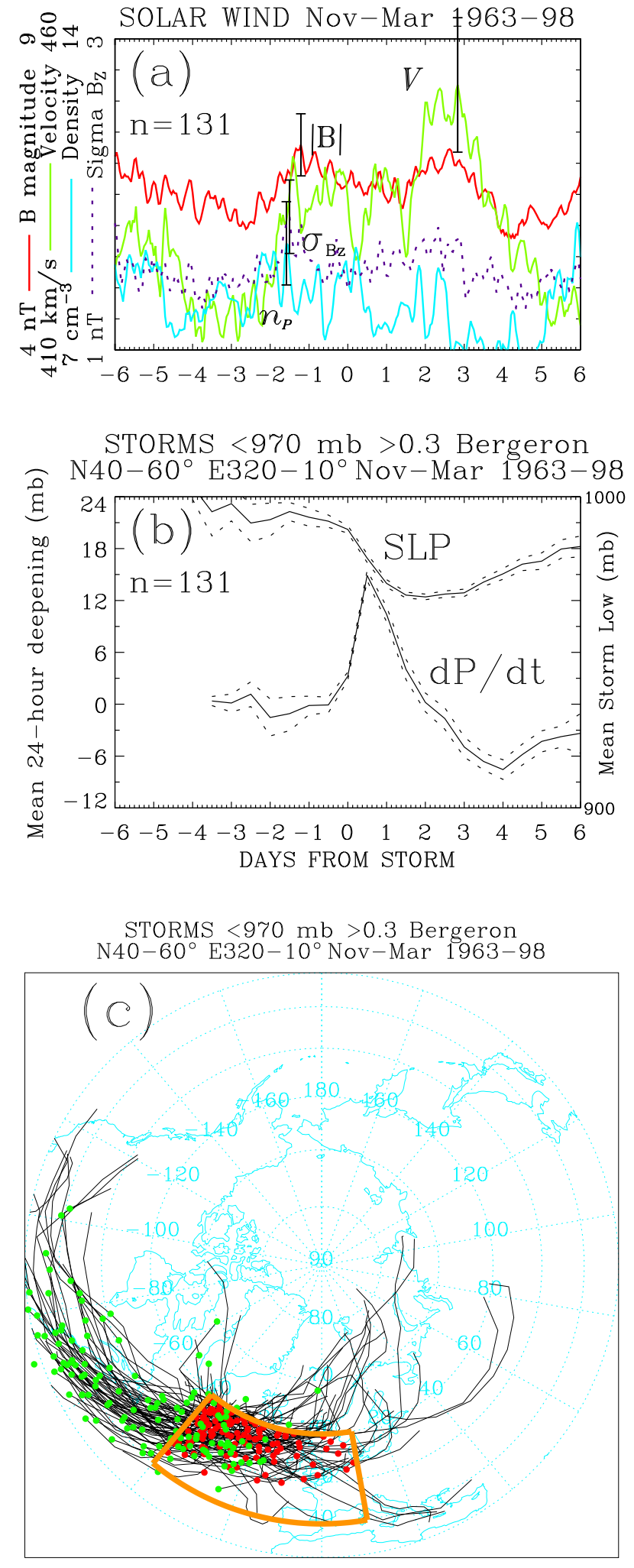

Fig. 14. The same as Fig. 12 but for the storm sector including the east Atlantic and the west coast of Europe. 

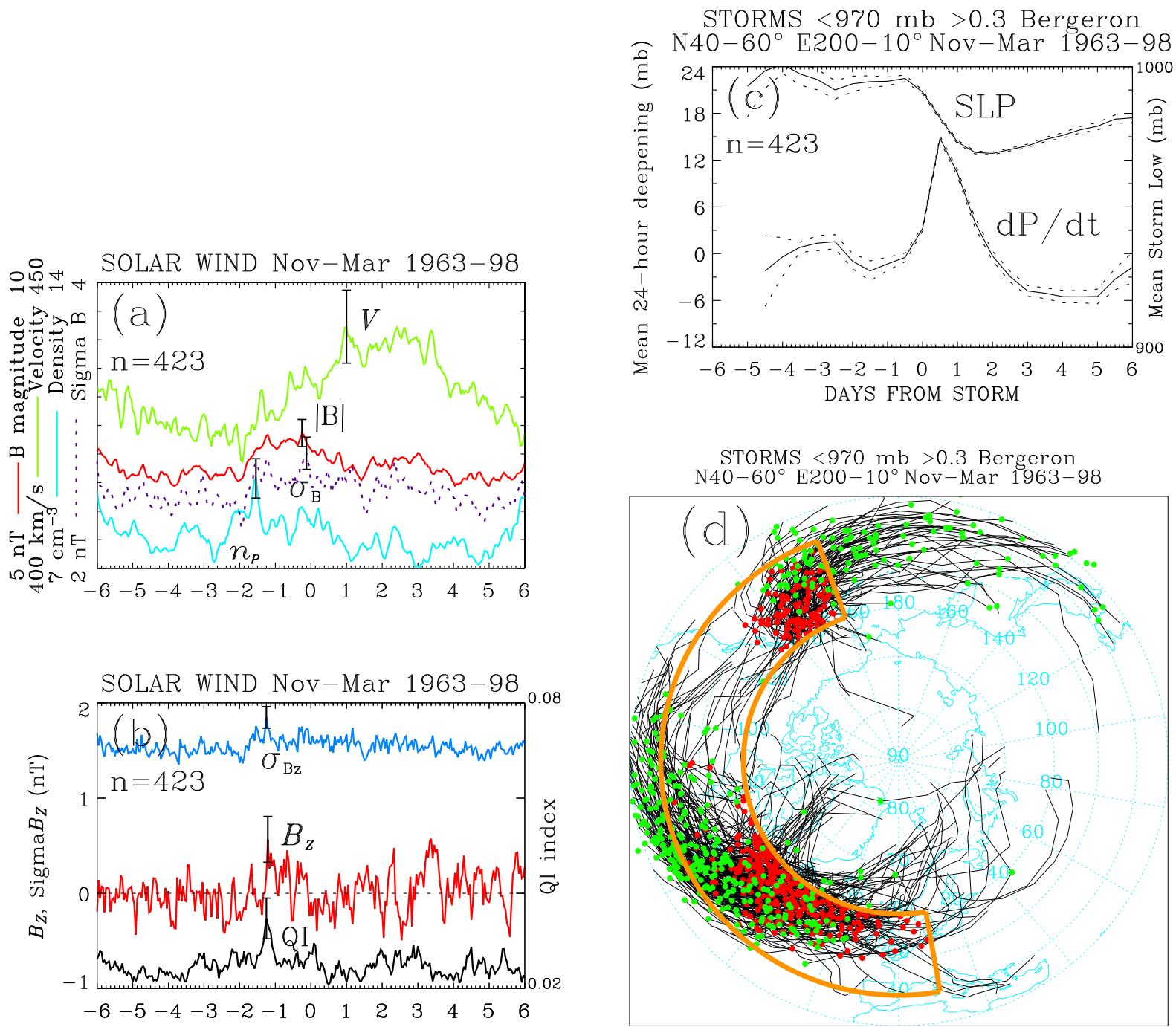

Fig. 15. The superposed epoch analysis of (a-b) solar wind plasma parameters and (c) the storm SLP minima and the deepening rate for the north-eastern Pacific storms. The time series are keyed by the start time of storms. (d) Storm tracks (black), locations of the storm first deepening greater than 0.3 Bergeron (green), and the storm lowest SLP (red) that was reached within the geographic sector (orange) that includes all three storm sectors, are shown.

arrival of high-speed solar wind is observed through years of high and low volcanic aerosol. This is consistent with the observed influence of solar wind on upper-level tropospheric vorticity and cloud band formation (Prikryl et al., 2009).

The results are discussed in the context of the AGW mechanism as well as the AEC model, which requires the presence of stratospheric aerosols for a significant Wilcox effect. The role of aerosols in the AEC mechanism is questioned but, in principle, the AGW and AEC mechanisms could operate in synergy, provided that the AEC model dependence on aerosols is not as restrictive as previously thought.

\section{Conclusions}

The results by Wilcox et al. (1974, 1976) showed a link between sector boundary passage and extratropical vorticity area index (VAI), namely a minimum in VAI one day after solar magnetic sector boundary passage by the Earth followed by an increase a few days later. These results are verified using updated data of the solar wind and green corona ( $\mathrm{Fe}$ XIV, $530.3 \mathrm{~nm}$ ), and the ERA-40 re-analysis of upper-level tropospheric vorticity at $300-$ and $500-\mathrm{mb}$ levels. The superposed epoch analysis of time series of VAI for latitudes $20^{\circ}-80^{\circ}$ keyed by the magnetic sector boundary passage during the northern and southern winters is extended for interval 
from 1963 to 2002. A one-to-one correspondence between quasi-periodic variations of the mean VAI and coronal intensity at low heliographic latitudes, coronal holes in particular, is found. The corresponding superposed epoch (SPE) analysis of solar wind parameters shows that recurrent streams from coronal holes modulate the VAI with a mean delay time of 1-2 days. The sector boundary crossings occur near the corotating stream interfaces, which are preceded by uncompressed slow solar wind that produces geomagnetically calm intervals, and which are followed by disturbed intervals due to the compressed solar wind at the leading edge of fast solar wind streams. The FFT spectra of VAI time series show peaks near the synodic solar rotation period and integral fractions of it that are similar to those found in the solar corona and solar wind.

With the exception of the Northern Hemisphere 500mb result after 1973 during years of low volcanic aerosol, namely the missing VAI minimum near day +1 , comparable and statistically significant amplitudes of the Wilcox effect are found during years of low and high volcanic aerosol loading. The IMF $B_{Z}$ seems to control the phase of the VAI response to sector boundary passage and the depth of the VAI minimum. For events associated with the north-to-south reversal of $B_{Z}$ the VAI response leads by up to 2 days the response to events associated with the south-to-north reversal of $B_{Z}$. For the latter, less geoeffective events, the VAI minimum deepens and the VAI maximum is delayed. The proposed mechanism that invokes auroral gravity waves (Prikryl et al., 2009) is consistent with these results. The atmospheric electrical current (AEC) mechanism (Tinsley et al., 1994) also may work provided there is less stress on the requirement of stratospheric aerosols for a significant effect (Tinsley and Zhou, 2006).

The VAI is an index of extratropical storminess. The observed variation of the mean VAI is consistent with a delayed response to quasi-periodic solar wind forcing of the atmosphere, a forcing which also generates auroral atmospheric gravity waves. On average, solar wind streams reach their maximum velocity 2 days after the arrival of corotating interaction regions (CIRs) while the solar wind magnetohydrodynamic waves have their largest amplitudes for about 2 days after the arrival of CIRs. It is suggested that the auroral gravity waves release instabilities such as the moist symmetric instability, leading to convection and latent heat release in extratropical cyclones. It is observed that explosive cyclogenesis and major extratropical storms including wind storms tend to occur within 2-3 days of the arrival of solar wind CIRs or coronal mass ejections. This conclusion is supported by the results of statistical analysis of an extensive database of extratropical storms showing that significant sea level pressure deepenings of mid-latitude storms tend to occur within a few days of the arrival of high-speed solar wind.

Acknowledgements. The ERA-40 dataset was provided by the European Centre for Medium-Range Weather Forecasts. The contri- butions by the ACE, IMP-8, SoHO, and Wind teams and OMNI project are acknowledged. The list of significant wind events in the area of Boulder, Colorado was obtained from a NOAA website and the Atlas of Extratropical Storm Tracks is available from a NASA website. We acknowledge contribution by Leif Svalgaard who kindly provided data of the interplanetary magnetic field polarity inferred from ground-based magnetometers. Valuable critical comments and suggestions were made by one of the reviewers. A part of this work was supported by the Slovak Research and Development Agency under the contract APVV-51-053805 and by the Science and Technology Assistance Agency under the contract APVT-51-012704.

Topical Editor M. Pinnock thanks one anonymous referee for her/his help in evaluating this paper.

\section{References}

Agee, E., Church, C. Morris, C., and Snow, J.: Some Synoptic Aspects and Dynamic Features of Vortices Associated with the Tornado Outbreak of 3 April 1974, Mon. Weather Rev., 103, 318333, 1975.

Ambrož, P.: Statistical method of superposition of epochs, I. Methodical analysis and some criteria of application, B. Astron. Inst. Czechosl., 30, 114-121, 1979.

Arora, B. R. and Padgaonkar, A. D.: Time-variation of solar influence on tropospheric circulation., J. Atmos. Terr. Phys., 43, 91-95, 1981.

Association of British Insurers (ABI): The vulnerability of UK property to windstorm damage, ABI Report, 1-47, 1981, 2003.

Balasubramanian, G. and Yau, M. K.: Baroclinic instability in a two-layer model with parameterized slantwise convection, J. Atmos. Sci., 51, 971-990, 1994a.

Balasubramanian, G. and Yau, M. K.: The effects of convection on a simulated marine cyclone, J. Atmos. Sci., 51, 2397-2417, 1994b.

Balasubramanian, G. and Yau, M. K.: Explosive marine cyclogenesis in a three-layer model with a representation of slantwise convection: A sensitivity study, J. Atmos. Sci., 52, 533-550, 1995.

Belcher, J. W. and Davis Jr., L.: Large-amplitude Alfvén waves in the interplanetary medium, 2, J. Geophys. Res., 76, 3534-3563, 1971.

Bhatnagar, V. P. and Jakobsson, T.: Lack of effects of solar magnetoc sector crossings on the troposphere, Geophys. Res. Lett., 5(3), 180-182, 1978.

Bolzan, M. J. A., Rosa, R. R., Ramos, F. M., Fagundes, P. R., and Sahai, Y.: Generalized thermostatistics and wavelet analysis of the solar Wind and proton density variability, J. Atmos. SolarTerr. Phys., 67(17-18), 1843-1851, 2005.

Borovsky, J. E. and Steinberg, J. T.: The "calm before the storm" in CIR/magnetosphere interactions: Occurrence statistics, solar wind statistics, and magnetospheric preconditioning, J. Geophys. Res., 111, A07S10, doi:10.1029/2005JA011397, 2006.

Bosart, L. F.: The Presidents' Day snowstorm of 18-19 February 1979: A subsynoptic-scale event, Mon. Weather Rev., 109, 1542-1566, 1981.

Bresky, W. C. and Colucci, S. J.: A forecast and analyzed cyclogenesis event diagnosed with potential vorticity, Mon. Weather Rev., 124, 2227-2244, 1996. 
Burlaga, L. F.: Interplanetary stream interfaces, J. Geophys. Res., 79, 3717-3725, 1974.

Burns, G. B., Bond, F. R., and Cole, K. D.: An investigation of the southern hemisphere vorticity response to solar sector boundary crossings, J. Atmos. Terr. Phys., 42, 765-769, 1980.

Burns, G. B., Tinsley, B. A., Troshichev, O. A., FrankKamenetsky, A. V., and Bering, E. A.: Interplanetary magnetic field and atmospheric electric circuit influences on ground level pressure at Vostok, J. Geophys. Res., 112, D04103, doi:10.1029/2006JD007246, 2007.

Burt, S. D. and Mansfield, D. A.: The Great storm of 15-16 October 1987, Weather, 43, 90-108, 1988.

Carrera, M. L., Gyakum, J. R., and Zhang, D.-L.: A numerical case study of secondary marine cyclogenesis sensitivity to initial error and varying physical processes, Mon. Weather Rev., 127, 641660, 1999.

Carslaw, K. S., Harrison, R. G., and Kirkby, J.: Cosmic rays, clouds and climate, Science, 298, 1732-1737, 2002.

Chandler, M. and Jonas, J.: Atlas of extratropical storm tracks (1961-1998), Tech. Rep., NASA Goddard Institute for Space Studies, New York, NY, available at http://www.giss.nasa.gov/ data/stormtracks, 1999.

Davis, C. A. and Emanuel, K. A.: Observational evidence for influence of surface heat fluxes on rapid maritime cyclogenesis, Mon. Weather Rev., 116, 2649-2659, 1988.

Dickinson, M. J., Bosart, L. F., Bracken, W. E., Hakim, G. J., Schultz, D. M., Bedrick, M. A., and Tyle, K. R.: The March 1993 Superstorm cyclogenesis: Incipient phase synoptic- and convective-scale flow interaction and model performance, Mon. Weather Rev., 125, 3041-3072, 1997.

Dixon, R. S., Browning, K. A., and Shutts, G. J.: The mystery of striated cloud heads in satellite imagery, Atmos. Sci. Lett., 1(1), 1-13, 2000.

Feren, G.: The "striated delta" cloud system - A satellite imagery precursor to major cyclogenesis in the eastern Australianwestern Tasman Sea region, Weather and Forecasting, 10, 286309,1995

Gosling, J. T., Asbridge, J. R., Bame, S. J., and Feldman, W. C.: Solar wind stream interfaces, J. Geophys. Res., 83, 1401-1412, 1978.

Gyakum, J. R.: On the Evolution of the $Q E$ II Storm. I: Synoptic Aspects, Mon. Weather Rev., 111, 1137-1155, 1983.

Gyakum, J. R. and Danielson, R. E.: Analysis of Meteorological Precursors to Ordinary and Explosive Cyclogenesis in the Western North Pacific, Mon. Weather Rev., 128, 851-863, 2000.

Harrison, R. G. and Carslaw, K. S.: Ion-aerosol-cloud processes in the lower atmosphere, Rev. Geophys., 41(3), 1012, doi:10.1029/2002RG000114, 2003.

Haurwitz, M. W. and Brier, G. W.: A critique of the superposed epoch analysis method: Its application to solar-weather relations, Mon. Weather Rev., 109, 2074-2079, 1981.

Heyraud, C., Protat, A., and Lamaître, Y.: Mesoscale processes and energetics of fastex secondary cyclogenesis, Proceedings ERAD 2004, 94-98, 2004.

Hines, C. O. and Halevy, I.: On the reality and nature of a certain sun-weather correlation, J. Atmos. Sci., 34, 382-404, 1977.

Hoeksema, J. T., Wilcox, J. M., and Scherrer, P. H.: The structure of the heliospheric current sheet: 1978-1982, J. Geophys. Res., 88, 9910-9918, 1983.
Holton, J. R.: An introduction to dynamic meteorology, Academic Press, p. 92-97, 1992.

Jarvis, M. J.: Planetary wave trends in the lower thermosphere Evidence for 22-year solar modulation of the quasi 5-day wave, J. Atmos. Sol.-Terr. Phys., 68(17), 1902-1912, 2006.

Kirkland, M. W., Tinsley, B. A., and Hoeksema, J. T.: Are stratospheric aerosols the missing link between tropospheric vorticity and Earth transits of the heliospheric current sheet?, J. Geophys. Res., 101(D23), 29 689-29699, 1996.

Klawa, M. and Ulbrich, U.: A model for the estimation of storm losses and the identification of severe winter storms in Germany, Nat. Hazards Earth Syst. Sci., 3, 725-732, 2003, http://www.nat-hazards-earth-syst-sci.net/3/725/2003/.

Kniveton, D. R. and Tinsley, B. A.: Daily changes in cloud cover and Earth transits of the heliospheric current sheet, J. Geophys. Res., 109, D11201, doi:10.1029/2003JD004232, 2004.

Kristjánsson, J. E. and Thorsteinsson, S.: The structure and evolution of an explosive cyclone near Iceland, Tellus, 47 A, 656-670, 1995.

Kunkel, K. E., Pielke Jr., R. A., and Changnon, S. A.: Temporal Fluctuations in Weather and Climate Extremes That Cause Economic and Human Health Impacts: A Review, B. Am. Meteorol. Soc., 80(6), 1077-1098, 1999.

Kuo, Y.-H. and Low-Nam, S.: Prediction of nine explosive cyclones over the western Atlantic ocean with a regional model, Mon. Weather Rev., 18, 3-25, 1990.

Lemaître, Y., Protat, A., and Scialom, G.: Dynamics of a "bomb like" deepening secondary cyclone from airborne Doppler radar, Q. J. Roy. Meteorol. Soc., 125, 2797-2818, 1999.

Larsen, M. F. and Kelley, M. C.: A study of an observed and forecasted meteorological index and its relation to the interplanetary magnetic field, Geophys. Res. Lett., 4, 337-340, 1977.

Lundstedt, H.: Influence of interplanetary interaction regions on geomagnetic disturbances and tropospheric circulation, Planet. Space Sci., 32, 1541-1545, 1984.

MacCallum, E.: The Burns' day storm 25 January 1990, Weather, 45, 166-173, 1990.

Madden, R. A.: Further Evidence of Traveling Planetary Waves., J. Atmos. Solar-Terr. Phys., 35, 1605-1618, 1978.

McDonald, N. J. and Roberts, W. O.: Further evidence of a solar corpuscular influence on large scale circulation at $300 \mathrm{mb}$, J. Geophys. Res., 65, 529-534, 1960.

Miyoshi, Y. and Kataoka, R.: Flux enhancement of the outer radiation belt electrons after the arrival of stream interaction regions, J. Geophys. Res., 113, A03S09, doi:10.1029/2007JA012506, 2008.

Mlynczak, M. G., Martin-Torres, F. J., Mertensm C. J., Marshall, B. T., Thompson, R. E., Kozyra, J. U., Remsberg, E. E., Gordley, L. L., Russell III, J. M., and Woods, T.: Solar-terrestrial coupling evidenced by periodic behavior in geomagnetic indexes and the infrared energy budget of the thermosphere, Geophys. Res. Lett., 35, L05808, doi:10.1029/2007GL032620, 2008.

Osherovich, V. A., Fainberg, J., and Stone, R. G.: Solar wind quasiinvariant as a new index of solar activity, Geophys. Res. Lett., 26(16), 2597-2600, 1999.

Parker, E. N.: Extension of the solar corona into interplanetary space, J. Geophys. Res., 64, 1675-1681, 1959.

Prikryl, P.: Solar wind plasma streams, high-level clouds and extratropical storms, Eos Trans. AGU, 87(52), Fall Meet. Suppl., 
Abstract SA41B-1424, 2006.

Prikryl, P., Muldrew, D. B., André, D. A., Sofko, G. J., Ruohoniemi, J. M., and Greenwald, R. A.: Upper-atmospheric gravity waves and tropospheric clouds, Eos Trans. AGU, 82(47), Fall Meet. Suppl., Abstract SA41B-0721, 2001.

Prikryl, P., Muldrew, D. B., and Sofko, G. J.: High-speed solar wind, auroral electrojets and atmospheric gravity waves: A link to the Earth's atmosphere, in Proceedings of the ISCS 2003 Symposium: Solar Variability as an Input to the Earth's Environment, Tatranska Lomnica, ESA SP-535, 371-376, 2003.

Prikryl, P., Muldrew, D. B., Sofko, G. J., and Ruohoniemi, J. M.: Solar wind Alfvén waves: A source of pulsed ionospheric convection and atmospheric gravity waves, Ann. Geophys., 23, 401417, 2005,

http://www.ann-geophys.net/23/401/2005/.

Prikryl, P., Muldrew, D. B., and Sofko, G. J.: The influence of solar wind on extratropical cyclones - Part 2: A link mediated by auroral atmospheric gravity waves?, Ann. Geophys., 27, 31-57, 2009 ,

http://www.ann-geophys.net/27/31/2009/.

Richardson, I. G. and Cane, H. V.: Regions of abnormally low proton temperature in the solar wind (1965-1991) and their association with ejecta, J. Geophys. Res., 100, 23 397-23 412, 1995.

Reed, R. J. and Albright, M. D.: A case study of explosive cyclogenesis in the Eastern Pacific, Mon. Weather Rev., 114, 2297-2319, 1986.

Roberts, W. O. and Olson, R. H.: Geomagnetic storms and wintertime 300-mb trough development in the North Pacific-North America Area, J. Atmos. Sci., 30, 135-140, 1973.

Roldugin, V. C. and Tinsley, B. A.: Atmospheric transmission changes associated with solar wind - induced ionization variations, J. Atmos. Solar Terr. Phys., 66, 114-1149, 2004.

Rosenberg, R. L. and Coleman Jr., P. J.: Solar cycle-dependent north-south field configurations observed in solar wind interaction regions, J. Geophys. Res., 85, 3021-3032, 1980.

Rostoker, G. and Sharma, R. P.: Correlation of high latitude tropospheric pressure with the structure of the interplanetary magnetic field, Can. J. Phys., 58(2), 255-269, 1980.

Russell, C. T. and McPherron, R. L.: Semiannual variation of geomagnetic activity, J. Geophys. Res., 78(1), 92-108, 1973.

Rušin, V. and Rybanský, M.: The green corona and magnetic fields, Solar Phys., 207, 47-61, 2002.

Rybanský, M.: Coronal index of solar activity, B. Astron. Inst. Czechosl., 26, 367-377, 1975.

Rybanský, M., Rušin, V., and Minarovijech, M.: Coronal index of solar activity, Space Sci. Rev., 95, 227-234, 2001.

Rybanský, M., Rušin, V., Minarovjech, M., Klocok, L., and Cliver, E. W.: Reexamination of the coronal index of solar activity, J. Geophys. Res., 110, A08106, doi:10.1029/2005JA011146, 2005.

Sanders, F.: Explosive Cyclogenesis in the West-Central North Atlantic Ocean, 1981-84. Part I: Composite Structure and Mean Behavior, Mon. Weather Rev., 114, 1781-1794, 1986.

Schultz, D. M. and Schumacher, P. N.: The use and misuse of conditional symmetric instability. Mon. Weather Rev., 127, 27092732, 1999.

Shapiro, R.: Solar magnetic sector structure and terrestrial atmospheric vorticity, J. Atmos. Sci., 33, 865-870, 1976.

Siscoe, G. L.: Structure and orientation of solar wind interaction fronts, J. Geophys. Res., 77, 27-34, 1972.
Svalgaard, L.: On the use of Godhavn $H$ component as an indicator of the interplanetary sector polarity, J. Geophys. Res., 80, 2717$2722,1975$.

Taylor Jr., H. A.: Selective factors in sun-weather research, Rev. Geophys., 24, 329-348, 1986.

Thorpe, A. J.: Extratropical cyclones An historic perspective, in Meteorology at the Millennium, pp. 14-22, Academic Press, London, 2002.

Tinsley, B. A.: Influence of solar wind on the global electric circuit, and inferred effects on cloud microphysics, temperature, and dynamics in the troposphere, Space Sci. Rev., 94, 231-258, 2000.

Tinsley, B. A. and Deen, G.: Apparent tropospheric response to $\mathrm{MeV}-\mathrm{GeV}$ particle flux variations: a connection via electrofreezing of supercooled water in high level clouds?, J. Geophys. Res., 96, 22 283-22 296, 1991.

Tinsley, B. A., Brown, G. M., and Scherrer, P. H.: Solar variability influences on weather and climate: Possible connections through cosmic ray fluxes and storm intensification, J. Geophys. Res., 94, 14 783-14 792, 1989.

Tinsley, B. A., Hoeksema, J. T., and Baker, D. N.: Stratospheric volcanic aerosols and changes in air-earth current density at solar wind magnetic sector boundaries as conditions for Wilcox tropospheric vorticity effect, J. Geophys. Res., 99(D8), 1680516813, 1994.

Tinsley, B. A. and Zhou, L.: Initial results of a global circuit model with stratospheric and tropospheric aerosols, J. Geophys. Res., 111, D16205, doi:10.1029/2005JD006988, 2006.

Tinsley, B. A., Rohrbaugh, R. P., and Hei, M.: Electroscavenging in clouds with broad droplet size distributions and weak electrification, Atmos. Res., 59-60, 115-136, 2001.

Tinsley, B. A., Zhou, L., and Plemmons, A.: Changes in scavenging of particles by droplets due to weak electrification in clouds, Atmos. Res., 79, 266-295, 2006.

Tinsley, B. A., Burns, G. B., and Zhou, L.: the role of the global circuit in solar and internal forcing of clouds and climate, Adv. Space Res., 40, 1126-1139, 2007.

Tsurutani, B. T. and Gonzalez, W. D.: The cause of high intensity long-duration continuous AE activity (HILDCAAs): Interplanetary Alfvén wave trains, Planet. Space Sci., 35, 405-412, 1987.

Uppala, S. M., Kållberg, P. W., Simmons, A. J., Andrae, U., da Costa Bechtold, V., Fiorino, M., Gibson, J. K., Haseler, J., Hernandez, A., Kelly, G. A., Li, X., Onogi, K., Saarinen, S., Sokka, N., Allan, R. P., Andersson, E., Arpe, K. , Balmaseda, M. A., Beljaars, A. C. M., van de Berg, L., Bidlot, J., Bormann, N., Caires, S., Chevallier, F., Dethof, A., Dragosavac, M., Fisher, M., Fuentes, M., Hagemann, S., Hólm, E., Hoskins, B. J., Isaksen, L., Janssen, P. A. E. M., Jenne, R., McNally, A. P., Mahfouf, J.-F., Morcrette, J.-J., Rayner, N. A., Saunders, R. W., Simon, P., Sterl, A., Trenberth, K. E., Untch, A., Vasiljevic, D., Viterbo, P., and Woollen, J.: The ERA-40 re-analysis, Q. J. Roy. Meteorol. Soc., 131, 2961-3012, 2005.

Wernli, H., Dirren, S., Liniger, M. A., and Zillig, M.: Dynamical aspects of the life cycle of the winter storm 'Lothar' (24-26 December 1999), Q. J. Roy. Meteorol. Soc., 128, 405-430, 2002.

Wilcox, J. M., Scherrer, P. H., Svalgaard, L., Roberts, W. O., and Olson, R. H.: Solar magnetic sector structure: Relation to circulation of the earth's atmosphere, Science, 180, 185-186, 1973.

Wilcox, J. M., Scherrer, P. H., Svalgaard, L., Roberts, W. O., Olson, R. H., and Jenne, R. L.: Influence of solar magnetic sector 
structure on terrestrial atmospheric vorticity, J. Atmos. Sci., 31, 581-588, 1974.

Wilcox, J. M., Svalgaard, L., and Scherrer, P. H.: Seasonal variation and magnitude of the solar sector structure - atmospheric vorticity effect, Nature, 255, 539-540, 1975.

Wilcox, J. M., Svalgaard, L., and Scherrer, P. H.: On the reality of a sun-weather effect, J. Atmos. Sci., 255, 1113-1116, 1976.

Williams, R. G.: A study of the energetics of a particular sunweather relation, Geophys. Res. Lett., 5(6), 519-522, 1978.
Williams, R. G. and Gerety, E. J.: Does the troposphere respond to day-to-day changes in solar magnetic field?, Nature, 275, 200201, 1978.

Zhou, L. and Tinsley, B. A.: Production of space charge at the boundaries of layer clouds, J. Geophys. Res., 112, D11203, doi:10.1029/2006JD007998, 2007.

Yu, F. and Turco, R. P.: From molecular clusters to nanoparticles: The role of ambient ionization in tropospheric aerosol formation, J. Geophys. Res., 106, 4797-4814, 2001. 